\title{
Gravitationally Lensed Quasar SDSS J1442+4055: Redshifts of Lensing Galaxies, Time Delay, Microlensing Variability, and Intervening Metal System at $z \sim 2$
}

\author{
Vyacheslav N. Shalyapin ${ }^{1,2,3}$ and Luis J. Goicoechea ${ }^{2}$ (D) \\ ${ }^{1}$ O.Ya. Usikov Institute for Radiophysics and Electronics National Academy of Sciences of Ukraine 12 Acad. Proscury St., 61085 Kharkiv, Ukraine; vshal@ukr.net \\ ${ }^{2}$ Departamento de Física Moderna Universidad de Cantabria Avda. de Los Castros s/n, E-39005 Santander, Spain; goicol@unican.es \\ Received 2018 December 15; revised 2019 February 9; accepted 2019 February 19; published 2019 March 12
}

\begin{abstract}
We present $r$-band photometric monitoring of the two images, A and B, of the gravitationally lensed quasar SDSS J1442+4055 using the Liverpool Telescope (LT). From the LT light curves between 2015 December and 2018 August, we derive at once a time delay of $25.0 \pm 1.5$ days ( $1 \sigma$ confidence interval; A is leading) and microlensing magnification gradients below $10^{-4}$ mag day ${ }^{-1}$. The delay interval is not expected to be affected by an appreciable microlensing-induced bias, so it can be used to estimate cosmological parameters. This paper also focuses on new Gran Telescopio Canarias (GTC) and LT spectroscopic observations of the lens system. We determine the redshift of two bright galaxies around the doubly imaged quasar using LT spectroscopy, while GTC data lead to low-noise individual spectra of A, B, and the main lensing galaxy, G1. The G1 spectral shape is accurately matched to an early-type galaxy template at $z=0.284$, and it has potential for further relevant studies. Additionally, the quasar spectra show absorption by metal-rich gas at $z \sim 2$. This dusty absorber is responsible for an extinction bump at a rest-frame wavelength of $2209 \pm 2 \AA$, which has strengths of $\sim 0.47$ and $0.76 \mathrm{mag}_{\mu} \mathrm{m}^{-1}$ for A and B, respectively. In such an intervening system, the dust-to-gas ratio, gas-phase metallicity indicator $[\mathrm{Zn} / \mathrm{H}]$, and dust depletion level $[\mathrm{Fe} / \mathrm{Zn}]$ are relatively high.
\end{abstract}

Key words: galaxies: high-redshift - gravitational lensing: strong - quasars: individual (SDSS J1442+4055)

Supporting material: machine-readable tables

\section{Introduction}

Gravitationally lensed quasars are becoming essential tools to study the structure and composition of galaxies at different redshifts and of the entire universe (Schneider et al. 2006; Treu 2010). Hence, significant effort is being devoted to the discovery of lensed quasars and their follow-up observations. For example, the current archive of the Sloan Digital Sky Survey (SDSS; York et al. 2000) includes photometric and spectroscopic data of more than 500,000 quasars (Pâris et al. 2018). The SDSS database contains a large collection of quasar spectra taken as part of the Baryon Oscillation Spectroscopic Survey (BOSS; Dawson et al. 2013), and More et al. (2016) took advantage of this fact to find 13 new double quasars. Other ongoing projects are also reporting discoveries of double/quadruple quasars and lists of lensed quasar candidates (e.g., Anguita et al. 2018; Kostrzewa-Rutkowska et al. 2018; Lemon et al. 2018). In addition, new multiple quasars must be fully characterized by follow-up observations. Detailed spectroscopy is used to identify intervening objects, analyze their gas, dust, and stellar content, and put constraints on the size and structure of sources through microlensing-induced spectral distortions (e.g., Wucknitz et al. 2003; Sluse et al. 2007; Mediavilla et al. 2011; Goicoechea \& Shalyapin 2016). Light curves of lensed quasars are also key pieces to determine time delays and constrain cosmological parameters (e.g., Vuissoz et al. 2008; Bonvin et al. 2017; Shalyapin \& Goicoechea 2017) and/or detect microlensing variability and thus learn about the structure of quasars and the composition of lensing galaxies (e.g., Kochanek 2004; Sluse et al. 2011; Hainline et al. 2013).

\footnotetext{
Corresponding author.
}

After performing data mining to identify double-quasar candidates in the SDSS-III DR10 (Ahn et al. 2014; Pâris et al. 2014), complementary observations of some of these candidates allowed Sergeyev et al. (2016) to find the new optically bright, wide-separation double quasar SDSS J1442+4055 (see also More et al. 2016). From a medium-resolution SDSS-BOSS spectrum of the A image of SDSS J1442+4055, Pâris et al. (2014) presented several estimates of the source (quasar) redshift. While the SDSS pipeline produced $z_{\mathrm{s}}=2.5746 \pm 0.0002$, a principal component analysis led to $z_{\mathrm{s}}=2.5931 \pm 0.0006$. After a visual inspection, Pâris et al. (2014) indicated that $z_{\mathrm{s}}=2.593$, and we adopt this value throughout the paper. The lens system basically consists of two quasar images (A and B) having $r \sim 18-19$ mag and separated by 2 !" 156 , as well as the main lensing galaxy (G1; $r \sim 19.5 \mathrm{mag}$ ), located 1."38 from the A image, and two additional intervening objects, a secondary galaxy (G2; $r \sim 19.8 \mathrm{mag}$ ) in the vicinity of G1 and an absorber at $z=1.946$ (Sergeyev et al. 2016).

This paper is dedicated to describing and deeply analyzing follow-up observations of SDSS J1442+4055. In the framework of the Gravitational LENses and DArk MAtter (GLENDAMA) project (Gil-Merino et al. 2018), in Section 2, we present a $2.7 \mathrm{yr}$ photometric monitoring with the $2.0 \mathrm{~m}$ Liverpool Telescope (LT) in the $r$ band and associated light curves for both quasar images, as well as spectroscopic observations with the $10.4 \mathrm{~m}$ Gran Telescopio Canarias (GTC) and the LT. The high signal-to-noise ratio $(\mathrm{S} / \mathrm{N})$ spectroscopy over a wide wavelength interval with the GTC allows us to accurately identify the primary lensing galaxy, G1, while we use the LT spectroscopic data of two bright secondary galaxies (G2 and another object in the field around the quasar) to measure their redshifts. In Section 3, the cross-correlation between the LT light curves of A and B yields the time delay in 


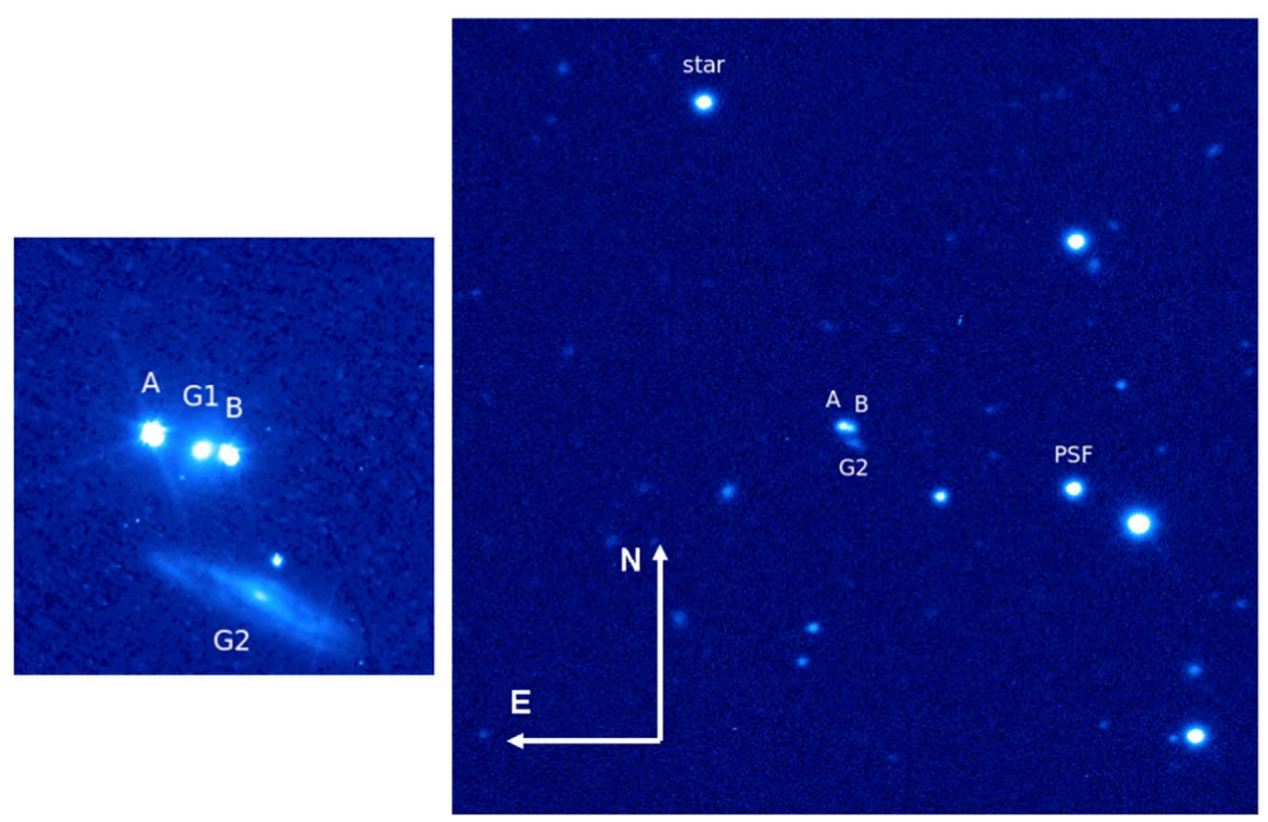

Figure 1. HST and LT imaging of SDSS J1442+4055. Left: HST-WFC3-G280 zeroth-order frame of size $10^{\prime \prime} \times 10^{\prime \prime}$. Right: first LT-IO:O $r$-band frame on 2015 December 22. This covers a field of view of $200^{\prime \prime} \times 200^{\prime \prime}$ and includes the PSF star and the control star (see main text).

the lens system. The $r$-band microlensing variability is also discussed in Section 3. In Section 4, using the GTC spectra of $\mathrm{A}$ and $\mathrm{B}$, we reveal the origin of the flux ratios $B / A$ from nearUV to near-IR. In Section 5, we study in detail the intervening gas at $z \sim 2$ and its connection with dust at the same redshift. In addition to the GTC and SDSS-BOSS spectra, this analysis is also based on data from the MMT Observatory, i.e., the medium-resolution UV-visible spectra (blueward of 6000 A) of the two quasar images with moderate $\mathrm{S} / \mathrm{N}$ (Findlay et al. 2018). Our main results and conclusions are summarized in Section 6.

When we were completing our third monitoring season and this work, Krogager et al. (2018) presented and analyzed Keck spectra of SDSS J1442+4055. In this paper, their spectroscopic results are compared to ours.

\section{Observations and Data Reduction}

\subsection{LT-IO:O Photometric Monitoring}

We have monitored the double quasar SDSS J1442+4055 over three complete observing seasons from 2015 December 23 to 2018 August 28. This monitoring suffers from two visibility gaps of about 4 months, which are not important in determining the relatively short time delay between images (see Section 3 ). All photometric observations were made in the $r$ band with the IO:O camera on the LT. With $2 \times 2$ binning (pixel scale of 0 ."30), we obtained frames of the lensed quasar for 135 observing epochs (nights). For the first 7 nights, we took two consecutive exposures of $250 \mathrm{~s}$ each, while $4 \times 150 \mathrm{~s}$ exposures were obtained on every remaining night. Thus, we collected 526 individual frames of 150 or $250 \mathrm{~s}$. The LT pipeline performed a primary data reduction, including bias subtraction, overscan trimming, and flat-fielding. Additionally, we interpolated over bad pixels and removed cosmic rays.

In order to extract fluxes for the two quasar images, we used the IMFITFITS software (McLeod et al. 1998). From this tool, assuming reasonable brightness profiles for the lensing galaxies G1 and G2, one can determine the fluxes of A and B through
Table 1

LT-IO:O $r$-band Light Curves of SDSS J1442+4055AB

\begin{tabular}{lcccccc}
\hline \hline MJD-50,000 & $m_{\mathrm{A}}{ }^{\mathrm{a}}$ & $e m_{\mathrm{A}}{ }^{\mathrm{a}}$ & $m_{\mathrm{B}}{ }^{\mathrm{a}}$ & $e m_{\mathrm{B}}{ }^{\mathrm{a}}$ & $m_{\mathrm{S}}{ }^{\mathrm{a}, \mathrm{b}}$ & $e m_{\mathrm{S}}{ }^{\mathrm{a}, \mathrm{b}}$ \\
\hline $7,379.288$ & 18.076 & 0.005 & 19.008 & 0.009 & 15.998 & 0.004 \\
$7,398.293$ & 18.029 & 0.006 & 19.012 & 0.012 & 15.993 & 0.006 \\
$7,400.289$ & 18.022 & 0.006 & 19.018 & 0.011 & 16.000 & 0.005 \\
$7,406.279$ & 18.026 & 0.005 & 18.998 & 0.009 & 15.993 & 0.005 \\
$7,412.267$ & 18.034 & 0.007 & 18.982 & 0.012 & 15.994 & 0.006 \\
\hline
\end{tabular}

Notes.

a-SDSS magnitude.

${ }^{\mathrm{b}}$ We use $\mathrm{S}$ to denote the field (control) star.

(This table is available in its entirety in machine-readable form.)

point-spread function (PSF) fitting. The brightness of the main deflector G1 was modeled as a de Vaucouleurs profile (More et al. 2016), whereas we take an exponential profile to model the light distribution of the secondary lens, G2, since the Hubble Space Telescope (HST) archive includes an image of G2 showing the presence of a disk and spiral $\mathrm{arms}^{4}$ (see the left panel of Figure 1). Both profiles were then convolved with the empirical PSF from the close star at R.A. $(\mathrm{J} 2000)=220^{\circ} .706485$ and decl. $(\mathrm{J} 2000)=+40^{\circ} .922076(r=16.075 \mathrm{mag}$; see the right panel of Figure 1). This PSF star was also used to model the point-like sources A and B. The relative positions of B and G1 (with respect to A), as well as the ellipticity, orientation, and effective radius of G1, were taken from Sergeyev et al. (2016). In addition, the ellipticity and orientation of $\mathrm{G} 2$ were set to their values in the SDSS database. In a first iteration, the fluxes of $\mathrm{G} 1$ and $\mathrm{G} 2$ and the relative position and effective radius of $\mathrm{G} 2$ were estimated from the best frames in terms of $\mathrm{S} / \mathrm{N}$ and FWHM seeing. In a second iteration, we applied the code to all individual frames, allowing the position of $\mathrm{A}$, the background level, and the fluxes of A and B to be free. We also calculated PSF fluxes for a field (control) star that is located at R.A.

\footnotetext{
4 Program ID: 14127; PI: Michele Fumagalli.
} 


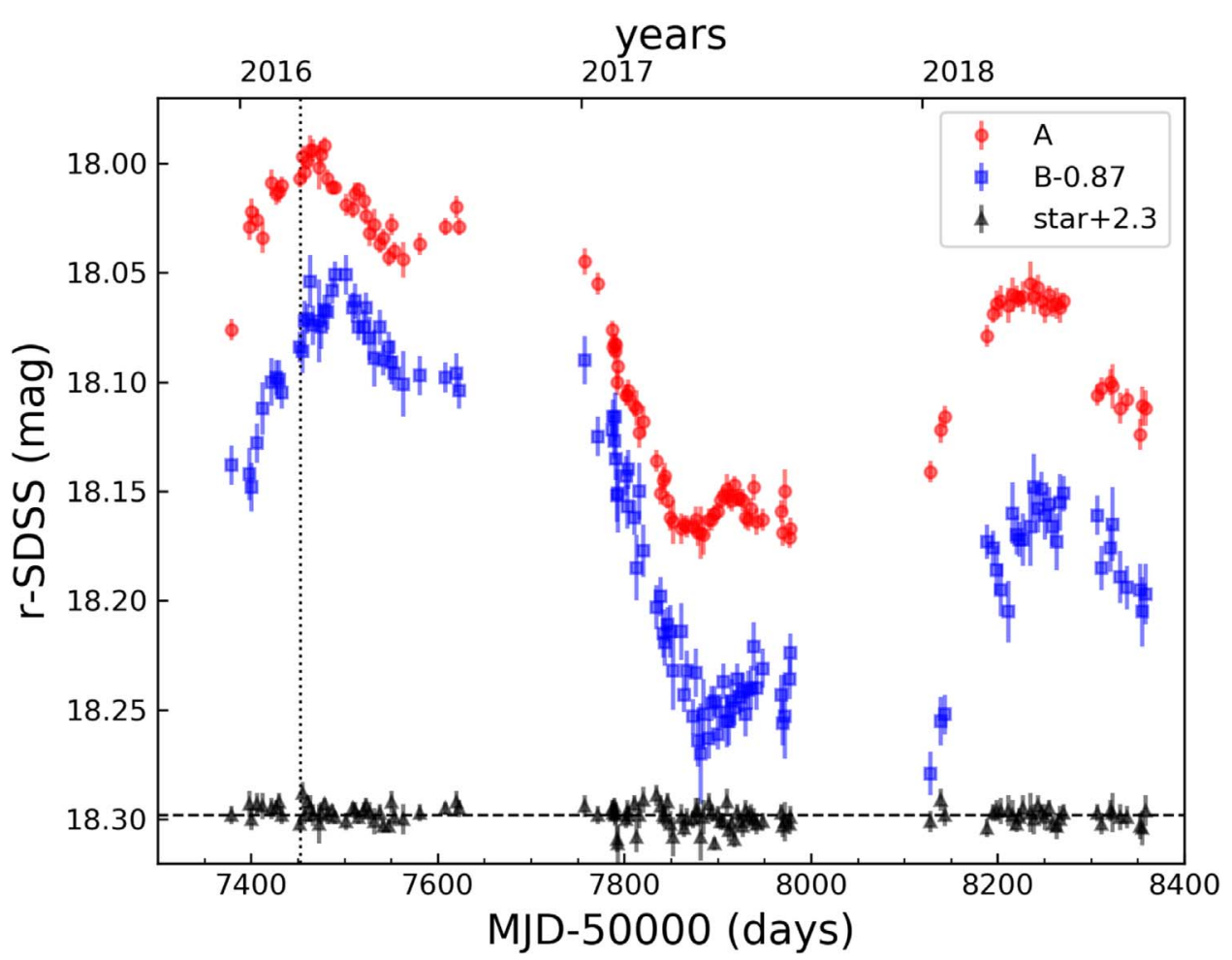

Figure 2. LT-IO:O $r$-band light curves of A, B, and the control star. The curves of B and the star are shifted by -0.87 and +2.3 mag, respectively, to facilitate comparison. The vertical dotted line corresponds to the epoch of our spectroscopic observations with GTC-OSIRIS (see Section 2.2), while the horizontal dashed line represents the constant flux of the control star.

$(\mathrm{J} 2000)=220^{\circ} .741396$ and decl. $(\mathrm{J} 2000)=+40^{\circ} .949647$ $(r=15.998$ mag; see the right panel of Figure 1).

The quasar light curves ( $r$-SDSS magnitudes) show anomalous results for 51 individual frames. These frames producing outliers are characterized by high FWHM seeing, low $\mathrm{S} / \mathrm{N}$ for $\mathrm{A}$, or tracking/guiding errors (very elongated or trailed stars); thus, we removed them from the final database. The remaining 475 frames represent $90 \%$ of the individual observations and have a median FWHM of 1 !' 28 . We then combined magnitudes measured on the same night to obtain final photometric data at 135 epochs. To estimate typical photometric errors in the light curves of $\mathrm{A}, \mathrm{B}$, and the control star, we used deviations between magnitudes having time separations $\leqslant 3$ days. This statistical analysis led to uncertainties of 0.0051 (A), 0.0093 (B), and 0.0046 (star) mag, which were multiplied by the relative $\mathrm{S} / \mathrm{N}$ at each epoch, $\langle\mathrm{S} / \mathrm{N}\rangle / \mathrm{S} / \mathrm{N}$, to calculate errors on a nightly basis $(\langle\mathrm{S} / \mathrm{N}\rangle$ is the average $\mathrm{S} / \mathrm{N}$; Howell 2006). Our final light curves of A, B, and the star are available in Table 1 and shown in Figure 2.

\subsection{GTC-OSIRIS Spectroscopy}

We performed spectroscopic observations of SDSS J1442 +4055 on 2016 March 5 using the OSIRIS instrument on the GTC. We took a $2850(3 \times 950)$ s GTC-OSIRIS exposure with each of two grisms, R500B and R500R, and used IRAF $^{5}$ packages to carry out data reductions. All $950 \mathrm{~s}$ subexposures were obtained in dark time, at low air masses, and under good

\footnotetext{
5 IRAF is distributed by the National Optical Astronomy Observatory, which is operated by the Association of Universities for Research in Astronomy (AURA) under cooperative agreement with the National Science Foundation. This software is available at http://iraf.noao.edu/.
}

seeing conditions. The average values of the air mass and the FWHM seeing at $6225 \AA$ amounted to 1.03 and $0 !$ " 89 , respectively. Regarding the dispersions, they were close to the nominal ones: $D(\mathrm{R} 500 \mathrm{~B})=3.560 \AA_{\text {pixel }}^{-1}$ and $D(\mathrm{R} 500 \mathrm{R})=$ $4.814 \AA \mathrm{Apixel}^{-1}$. However, we slightly modified the standard wavelength ranges, decreasing the minimum wavelength for R500B ( $3425 \AA$; to include relevant absorption features) and the maximum wavelength for R500R ( $9255 \AA$; to avoid fringing and second-order contamination). The spatial pixel scale was $0 . \prime 254$.

In order to extract spectra of all individual sources in the strong lensing region, the 1 !" 23 wide slit was oriented along the line joining $\mathrm{A}$ and $\mathrm{B}$, and we followed a technique similar to those in our previous analyses of GTC-OSIRIS spectroscopic data (e.g., Goicoechea \& Shalyapin 2016). We modeled the lens system as a $2 \mathrm{D}$ light distribution consisting of two pointlike sources (A and B) and a circular de Vaucouleurs profile with $r_{\text {eff }}=0$ ". 59 (G1), whose relative positions are given in Table 2 of Sergeyev et al. (2016). Such an ideal model was then convolved with a 2D Moffat PSF having a power index $\beta=3$, masked with the slit transmission, and integrated across the slit. Apart from the position of A and the FWHM value, our 1D model at each wavelength bin included the fluxes of A, B, and G1 as free parameters; thus, fits to the GTC-OSIRIS 1D data allowed us to obtain the spectra of the two quasar images and the main lensing galaxy. For each source, in addition to wavelength-dependent fluxes $F_{\lambda}$, we estimated flux errors $e F_{\lambda}$ using Equation (9) of Horne (1986). This method for extracting individual spectra is significantly different from the technique used by Krogager et al. (2018), who considered Keck-LRIS observations in the wavelength range $3600-8650 \AA$ on 2016 June 5, extracted data of A and B that are contaminated by light from $\mathrm{G} 1$, and then fitted templates for the intrinsic spectral 
Table 2

GTC-OSIRIS R500B Spectra of SDSS J1442+4055ABG1

\begin{tabular}{lcccrr}
\hline \hline$\lambda^{\mathrm{a}}$ & $F_{\lambda}(\mathrm{A})^{\mathrm{b}}$ & $e F_{\lambda}(\mathrm{A})^{\mathrm{c}}$ & $F_{\lambda}(\mathrm{B})^{\mathrm{b}}$ & $e F_{\lambda}(\mathrm{B})^{\mathrm{c}}$ & $F_{\lambda}(\mathrm{G} 1)^{\mathrm{b}}$ \\
\hline 3426.770 & 17.928 & 1.660 & 14.001 & 1.520 & -10.045 \\
3430.330 & 19.053 & 1.569 & 9.429 & 1.446 & -3.607 \\
3433.891 & 19.969 & 1.490 & 8.143 & 1.324 & 0.734 \\
3437.452 & 17.532 & 1.420 & 9.126 & 1.259 & -3.928 \\
3441.013 & 21.708 & 1.435 & 7.852 & 1.201 & -2.417 \\
\hline
\end{tabular}

Notes.

a Observed wavelength in $\AA$.

${ }^{\mathrm{b}}$ Flux in $10^{-17} \mathrm{erg} \mathrm{cm}^{-2} \mathrm{~s}^{-1} \AA^{-1}$.

c Flux error in $10^{-17} \mathrm{erg} \mathrm{cm}{ }^{-2} \mathrm{~s}^{-1} \AA^{-1}$.

(This table is available in its entirety in machine-readable form.)

Table 3

GTC-OSIRIS R500R Spectra of SDSS J1442+4055ABG1

\begin{tabular}{lccccc}
\hline \hline$\lambda^{\mathrm{a}}$ & $F_{\lambda}(\mathrm{A})^{\mathrm{b}}$ & $e F_{\lambda}(\mathrm{A})^{\mathrm{c}}$ & $F_{\lambda}(\mathrm{B})^{\mathrm{b}}$ & $e F_{\lambda}(\mathrm{B})^{\mathrm{c}}$ & $F_{\lambda}(\mathrm{G} 1)^{\mathrm{b}}$ \\
\hline 4846.734 & 22.387 & 0.362 & 9.399 & 0.274 & 4.743 \\
4851.550 & 22.515 & 0.358 & 9.669 & 0.271 & 4.009 \\
4856.365 & 23.775 & 0.353 & 9.587 & 0.265 & 0.305 \\
4861.180 & 24.849 & 0.346 & 10.300 & 0.261 & 0.300 \\
4865.995 & 23.896 & 0.322 & 10.484 & 0.243 & 0.293 \\
\hline
\end{tabular}

Notes.

a Observed wavelength in $\AA$.

b Flux in $10^{-17} \mathrm{erg} \mathrm{cm}^{-2} \mathrm{~s}^{-1} \AA^{-1}$.

${ }^{c}$ Flux error in $10^{-17} \mathrm{erg} \mathrm{cm}^{-2} \mathrm{~s}^{-1} \AA^{-1}$.

(This table is available in its entirety in machine-readable form.)

slopes of A, B, and G1 and the supposed reddening of A and B arising from dust in the absorber at $z=1.946$ (we justify this hypothesis in Section 4).

We also checked our wavelength and flux calibrations, which were based on $\mathrm{HgAr}$ and $\mathrm{Ne}$ arc lamp exposures, as well as spectra of the standard star Hilt 600 (Hamuy et al. 1992, 1994). First, we compared the positions of narrow absorption lines in the GTC-OSIRIS spectra of A and the SDSS-BOSS spectrum of the brightest quasar image, taken on 2012 May 29. This comparison allowed detection of systematic deviations in our wavelength zero-points, so the R500B and R500R data were shifted by +1.5 and $-2.0 \AA$, respectively. Second, we used $r$-band frames taken with the LT on 2016 March 4 to measure the $r$-band fluxes of A and B and compare them to the corresponding GTC-OSIRIS fluxes. These spectral fluxes agreed well with the LT photometry (typical deviation of $\sim 1 \%$ ), so the spectral energy distributions were not rescaled. The final calibrated spectra of A, B, and G1 are included in Tables 2 and 3. In addition, the results from the observations with both grisms are plotted in Figure 3. It is also worth mentioning that the spectral shapes of $\mathrm{A}$ and $\mathrm{B}$ in the wavelength range $3500-6000 \AA$ are consistent with those obtained with the HST-WFC3 G280 grism on 2016 April 21, i.e., about 7 weeks later (Lusso et al. 2018). All raw and reduced frames in FITS format are publicly available at the GTC archive. ${ }^{6}$

In the top panels of Figure 3, we show the R500B (left) and R500R (right) spectra of A (red), B (blue), and G1 (green). The accurate quasar spectra contain five prominent emission features at $z_{\mathrm{s}}=2.593-\mathrm{O}$ VI, Ly $\alpha, \mathrm{Si}$ IV/O IV], C IV, and

\footnotetext{
6 http://gtc.sdc.cab.inta-csic.es/gtc/index.jsp.
}

C III] (vertical dotted lines) - and enable us to probe flux ratios $B / A$ over a very broad interval of wavelengths from near-UV to near-IR (3430-9250 ̊). Furthermore, the GTC-OSIRIS spectra of A and B include an intervening metal system (IMS), which was also detected in the SDSS-BOSS spectrum of A (Sergeyev et al. 2016) and the Keck spectra of both quasar images (Krogager et al. 2018). We measured $z_{\mathrm{IMS}}=1.9465$ using strong Fe II/Mg II absorption lines in the SDSS-BOSS spectral energy distribution. The physical properties of this high- $z$ galaxy halo are widely discussed in Sections 4 and 5. We do not pay special attention to other absorbers. For example, there is a proximate system at $z=2.586 \sim z_{\mathrm{s}}$ consisting of neutral hydrogen ( $\operatorname{Ly} \alpha$ and $\operatorname{Ly} \beta$ lines) and high-ionization metals ( $\mathrm{OVI}, \mathrm{NV}$, Si IV, and C IV lines). This is most probably associated with the quasar host galaxy or its environment (e.g., Ellison et al. 2010). There are also prominent Ly $\alpha$ systems at $z=2.578,2.406$, and 2.296, so the HST-WFC3-G280 spectra blueward of $3300 \AA$ are strongly absorbed by neutral hydrogen. The Ly $\alpha$ break at $\lambda=3000 \mathrm{~A}$ (Lusso et al. 2018) is mainly due to the nearest $\operatorname{Ly} \alpha$ system at $z=2.296$.

In the bottom panels of Figure 3, we can appreciate details of the R500B (left) and R500R (right) spectra of the lensing galaxy G1 (green). The spectral shapes and the positions of several absorption features (vertical dashed lines; e.g., the Ca II HK doublet, $G$ band, $\mathrm{H} \beta$ line, and $\mathrm{Mg}$ I $b$ triplet) match very well with an early-type galaxy template ${ }^{7}$ at $z=0.284$ (magenta). Hence, $z_{\mathrm{G} 1}=0.284 \pm 0.001$ was inferred from the positions of the absorption lines. The G1 spectra and the templates in the bottom panels of Figure 3 are of a similar

\footnotetext{
7 SDSS spectral template No. 23 at http://classic.sdss.org/dr7/algorithms/ spectemplates/index.html.
} 

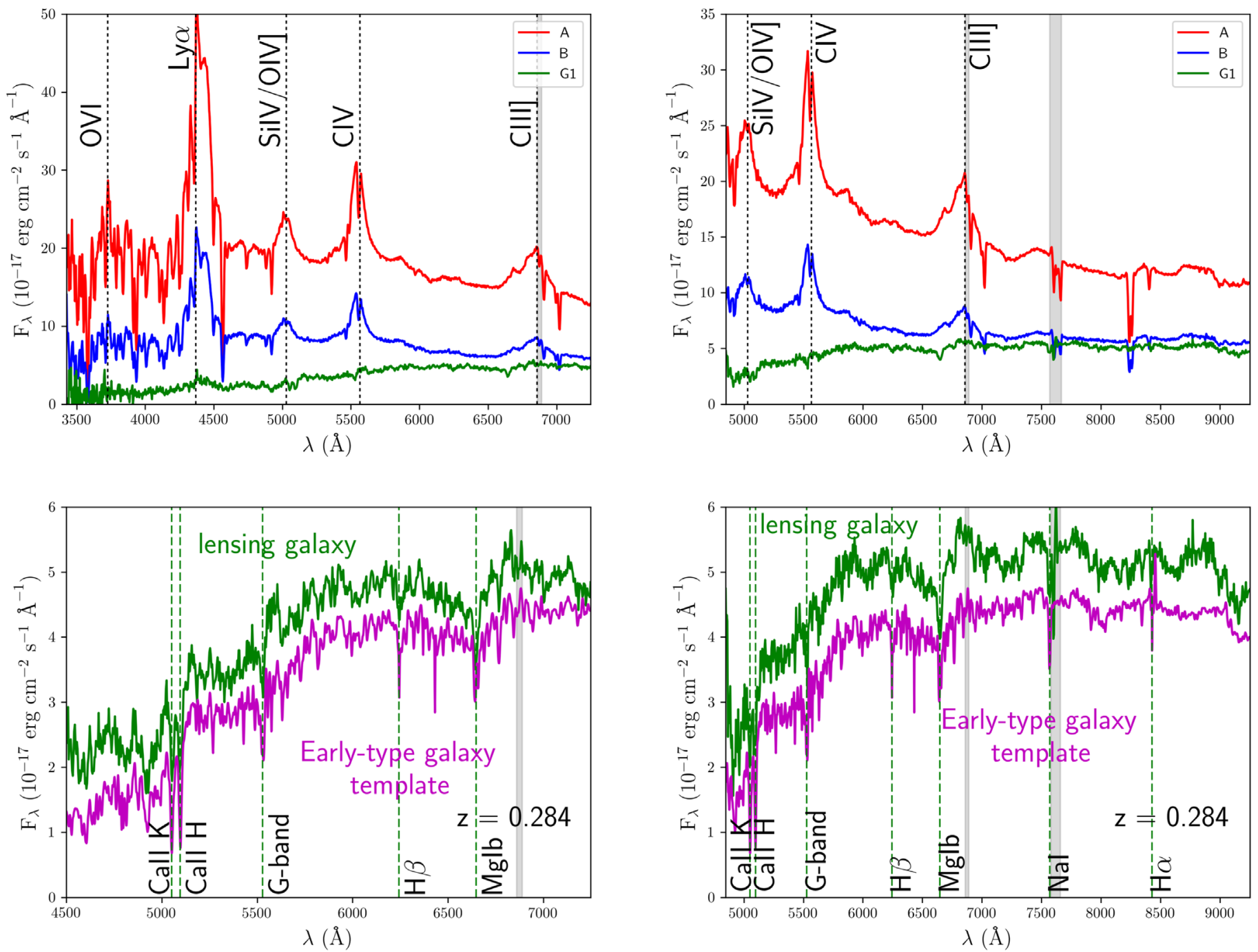

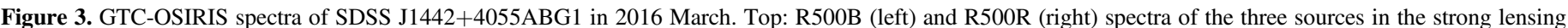

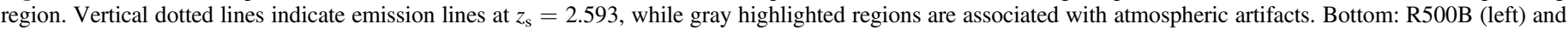

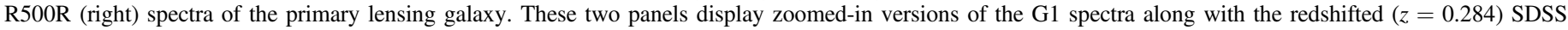
spectral template of an early-type galaxy. Vertical dashed lines are associated with absorption features.

quality, whereas the Keck-LRIS spectrum of G1 in Figure 3 of Krogager et al. (2018) is much noisier. In any case, our $z_{\mathrm{G} 1}$ value fully agrees with the lens redshift from Keck-LRIS data, which was based on a complex fit (see above). This consistency of results through different data sets and analysis techniques strengthens the reliability of the measured redshift.

\subsection{LT-SPRAT Data}

In the vicinity of the double quasar, there is a secondary lensing galaxy (G2) that is displayed in Figure 4 of Sergeyev et al. (2016). The two galaxies G1 and G2 are $<5$ " apart, so they could be physically associated. Indeed, these sources have a similar $r$-band brightness, and $z_{\mathrm{G} 1}$ (see Section 2.2) is consistent with the SDSS photometric redshift of G2: $0.323 \pm 0.051$. To identify G2 and another bright field galaxy (G3), both objects were spectroscopically observed on 2016 June 8. The SDSS position of G3 is R.A. $(\mathrm{J} 2000)=220^{\circ} .73908$ and decl. $(\mathrm{J} 2000)=+40^{\circ} .92188$ (southeast of the quasar images); thus, A and G3 are separated by 33". 9 . Moreover, G3 is brighter than $\mathrm{G} 2(r=19.1)$ and has a photometric redshift of $0.188 \pm 0.029$.
Table 4

LT-SPRAT Spectra of SDSS J1442+4055G2G3

\begin{tabular}{lrr}
\hline \hline$\lambda^{\mathrm{a}}$ & $F_{\lambda}(\mathrm{G} 2)^{\mathrm{b}}$ & $F_{\lambda}(\mathrm{G} 3)^{\mathrm{b}}$ \\
\hline 3984.359 & 6.368 & -5.175 \\
3988.991 & 12.435 & 4.964 \\
3993.623 & 3.922 & 3.153 \\
3998.255 & 4.809 & 2.718 \\
4002.887 & -6.239 & 4.339 \\
\hline
\end{tabular}

Notes.

a Observed wavelength in $\AA$.

${ }^{b}$ Flux in $10^{-17} \mathrm{erg} \mathrm{cm}^{-2} \mathrm{~s}^{-1} \AA^{-1}$.

(This table is available in its entirety in machine-readable form.)

We used the red grating mode of the SPRAT instrument on the LT, which is optimized for the red region of the 4000-8000 A wavelength range. Additionally, the 1." 8 wide slit was oriented along the line joining G2 and G3. The dispersion and spatial pixel scale were $4.63 \AA$ pixel $^{-1}$ and 0 ." 44 . We took $5 \times 600$ s science exposures under good observing conditions: moonless night, FWHM $\sim 1^{\prime \prime}$, and air mass of $\sim 1.1$. Tungsten 


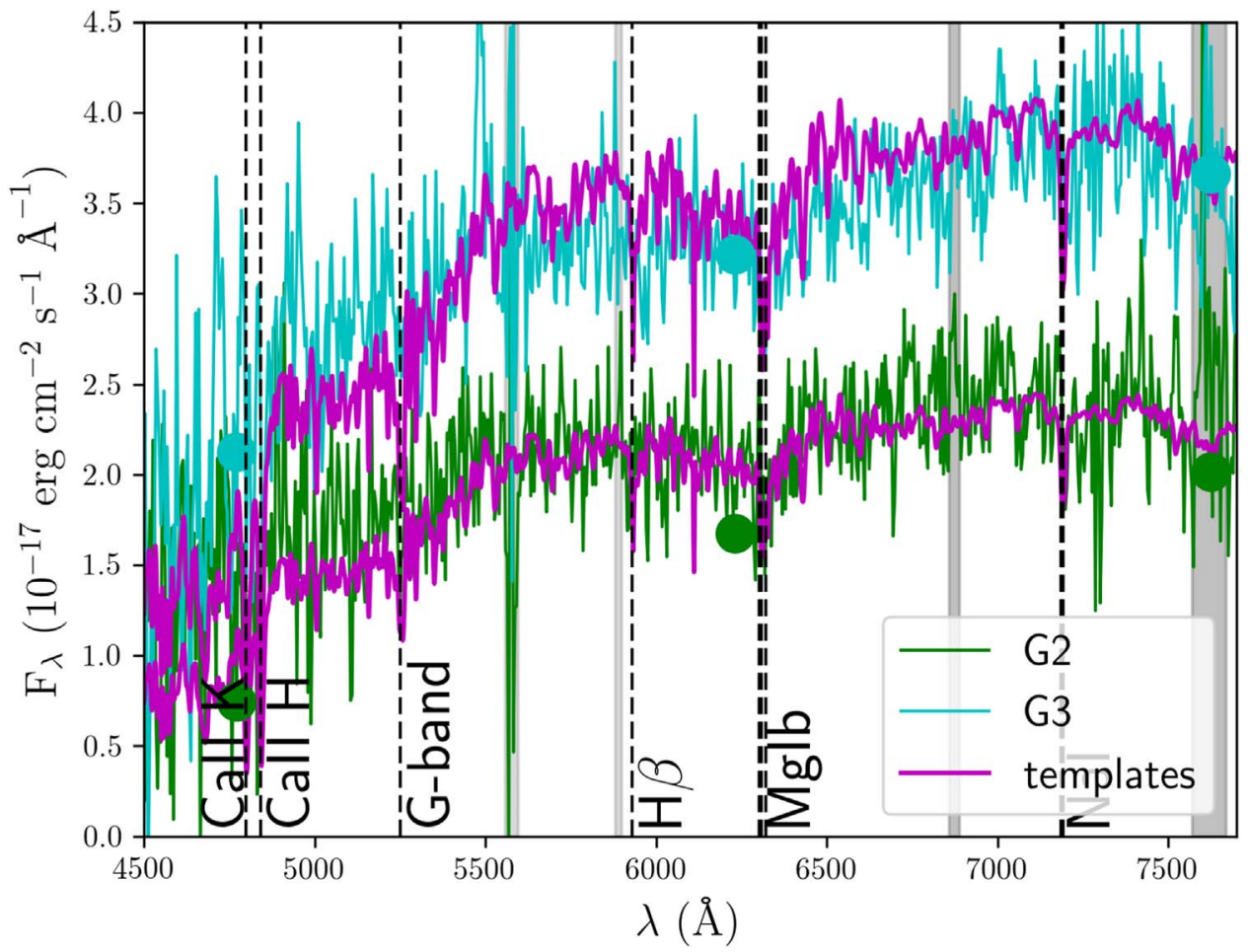

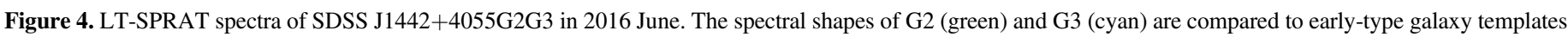

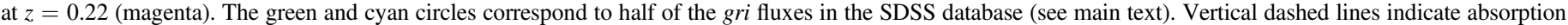
features at $z=0.22$, while gray highlighted regions are associated with atmospheric artifacts.

lamp and Xe arc exposures were used for flat-fielding and wavelength calibration, respectively. We also observed the spectrophotometric standard star BD+33d2642 (Oke 1990) for flux calibration. After a primary reduction of frames under the IRAF working environment, the spectra of G2 and G3 were extracted using the task APALL. These spectroscopic data are included in Table 4 and Figure 4.

In Figure 4, we show the two spectra (green and cyan) and two redshifted SDSS templates of an early-type galaxy (magenta; see Section 2.2), along with gri fluxes for both galaxies from the SDSS database (circles). Despite G2 being a spiral galaxy (see Section 2.1), we do not detect any emission lines in its visible spectrum. The original SDSS fluxes of G2 and G3 were reduced by $50 \%$ to roughly account for slit losses, since the slit width does not cover their entire luminous halo. Although the LT-SPRAT spectra are quite noisy, their shapes indicated that the two secondary galaxies are at similar redshift, $z_{\mathrm{G} 2}=z_{\mathrm{G} 3}=0.22 \pm 0.01$. Hence, the photometric redshift of $\mathrm{G} 2$ does not correspond to the true value of $z_{\mathrm{G} 2}$, and this galaxy is not physically associated with the main deflector.

\section{Time Delay and Microlensing Variability}

The quasar light curves in Figure 2 display almost parallel prominent variations, which suggest a short time delay between images and a slow microlensing signal. In this section, we use two standard techniques to measure the time delay, identify the microlensing variability, and thus confirm our qualitative conclusion. First, we considered the dispersion method to match both light curves-more precisely, the $D_{4,2}^{2}$ estimator (Pelt et al. 1996), including a step function-like (seasonal) microlensing. The value of the decorrelation length $(\delta)$ has little influence on the best solutions for the time delay $\left(\Delta t_{\mathrm{AB}}=\tau_{\mathrm{B}}-\tau_{\mathrm{A}}\right)$ and the three magnitude offsets (one per season; $\left.\Delta m_{\mathrm{AB}}=m_{\mathrm{B}}\left(t+\Delta t_{\mathrm{AB}}\right)-m_{\mathrm{A}}(t)\right)$, and after checking results for 4 days $\leqslant \delta \leqslant 20$ days, we chose an intermediate value of 10 days to estimate confidence intervals. We generated $10^{4}$ simulated light curves of each quasar image at epochs equal to those of the observation, modifying the observed magnitudes by adding random quantities (repetitions of the LT experiment). These additive random numbers were realizations of normal distributions around zero, with standard deviations equal to the measured uncertainties. The $D_{4,2}^{2}$ estimator ( $\delta=10$ days) with a step function-like microlensing was then applied to each pair (A and B) of simulated curves to produce distributions of delays and magnitude offsets. The delay histogram is shown in the top left panel of Figure 5.

Second, we carried out a reduced $\chi^{2}\left(\chi_{\mathrm{r}}^{2}\right)$ minimization with three magnitude offsets, i.e., considering a seasonal microlensing similar to that of the dispersion method. The $\chi_{\mathrm{r}}^{2}$ technique has two variants (e.g., Ullán et al. 2006): $\chi_{\mathrm{r}, \mathrm{A}}^{2}$ compares the curve $\mathrm{A}$ with the time-shifted and binned curve $\mathrm{B}$, and $\chi_{\mathrm{r}, \mathrm{B}}^{2}$ compares the curve $\mathrm{B}$ with the time-shifted and binned curve A. In both variants, bins are characterized by a semisize $\alpha$, which plays a role similar to the decorrelation length in $D_{4,2}^{2}$. Reasonable values of $\alpha$ (in the interval 4-20 days) led to similar best solutions for the delay and the magnitude offsets, so we focused on results for $\alpha=10$ days. It is also worth mentioning that the best solutions for $\alpha=10$ days correspond to $\chi_{\mathrm{r}, \mathrm{A}}^{2}=0.69$ and $\chi_{\mathrm{r}, \mathrm{B}}^{2}=0.91$. This means that the seasonal microlensing scenario works quite well, and more complex models (e.g., linear or quadratic microlensing variations) are not required. We performed $\chi_{\mathrm{r}, \mathrm{A}}^{2}$ and $\chi_{\mathrm{r}, \mathrm{B}}^{2}$ minimizations with a step function-like microlensing for the $10^{4}$ pairs of simulated curves, yielding delay and magnitude-offset distributions that appear in the top panels of Figure 5. The magnitude-offset 

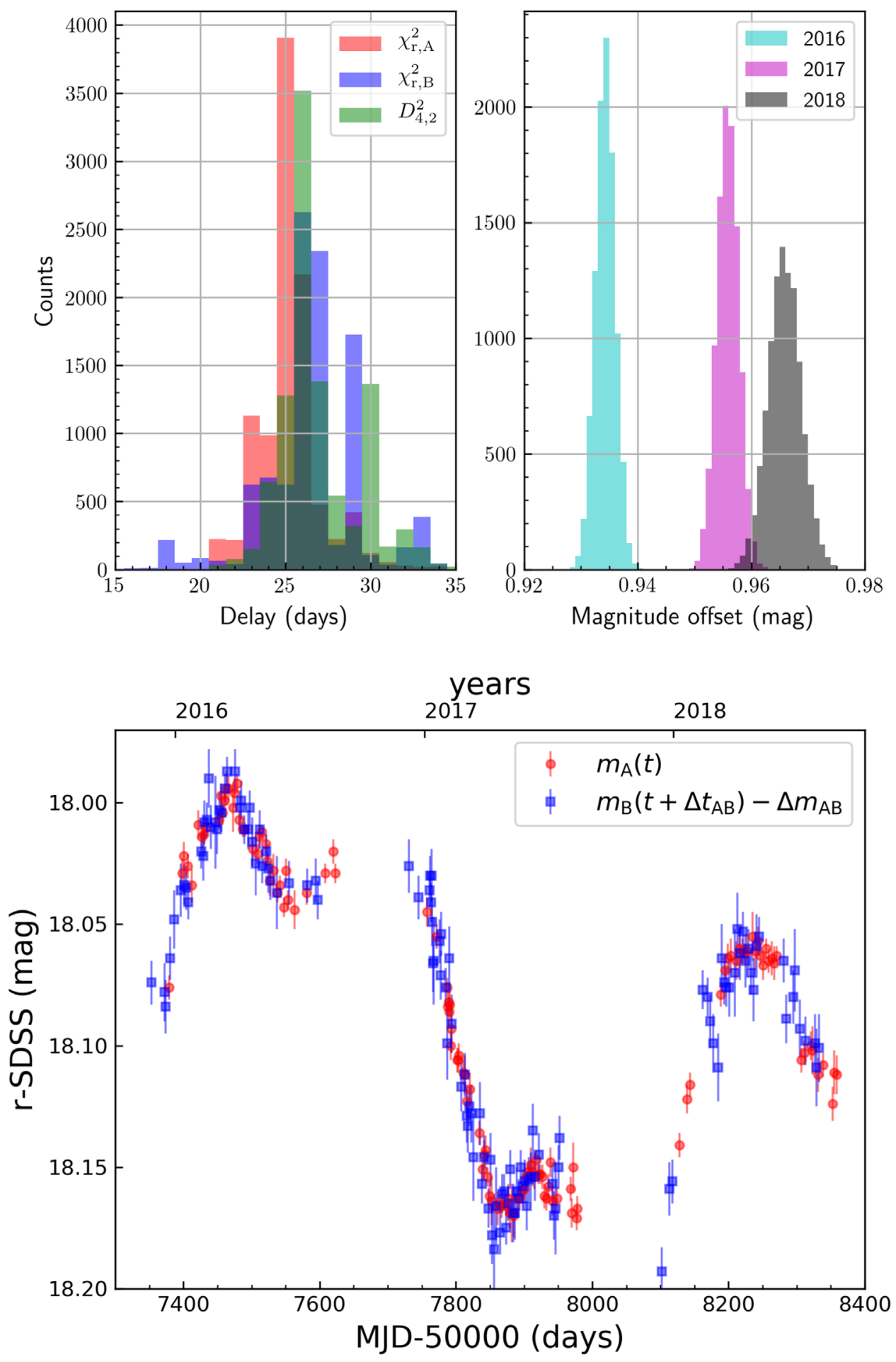

Figure 5. Top: histograms from $10^{4}$ pairs of simulated curves $\mathrm{AB}$. The left panel shows the best solutions of the time delay from the $D_{4,2}^{2}, \chi_{\mathrm{r}, \mathrm{A}}^{2}$, and $\chi_{\mathrm{r}, \mathrm{B}}^{2}$ estimators, including seasonal microlensing $\left(\delta=\alpha=10\right.$ days). The right panel displays the best solutions of the magnitude offsets in 2016, 2017, and 2018 from the $\chi_{\mathrm{r}, \mathrm{A}}^{2}$ minimization. Bottom: combined light curve in the $r$ band. The A curve is compared with the magnitude- and time-shifted $\mathrm{B}$ curve $\left(\Delta t_{\mathrm{AB}}=25\right.$ days, $\Delta m_{\mathrm{AB}}(2016)=0.934 \mathrm{mag}, \Delta m_{\mathrm{AB}}(2017)=0.956 \mathrm{mag}$, and $\Delta m_{\mathrm{AB}}(2018)=0.966 \mathrm{mag}$; see main text $)$.

histograms from the $D_{4,2}^{2}, \chi_{\mathrm{r}, \mathrm{A}}^{2}$, and $\chi_{\mathrm{r}, \mathrm{B}}^{2}$ estimators are practically identical; thus, indeed, we only include results from $\chi_{\mathrm{r}, \mathrm{A}}^{2}$ in the top right panel of Figure 5.

From the delay distributions in the top left panel of Figure 5, we obtained the $1 \sigma$ measurements (68\% confidence intervals) in Table 5. The $\chi_{\mathrm{r}, \mathrm{B}}^{2}$ and $D_{4,2}^{2}$ methods provide delay histograms incorporating a secondary peak at $29-30$ days, which is probably an artifact due to the use of the light curve of B as a template for variability $\left(\chi_{\mathrm{r}, \mathrm{B}}^{2}\right)$ or related to not differentiating between the role that $\mathrm{A}$ and B play $\left(D_{4,2}^{2}\right)$. Contrarily, the $\chi_{\mathrm{r}, \mathrm{A}}^{2}$ estimator does not provide significant signal at 29-30 days. This last technique relies on the use of the light curve of $\mathrm{A}$ as a reference template and the binned light curve of $\mathrm{B}$, and it is expected to yield the least-biased results (errors in A are about 

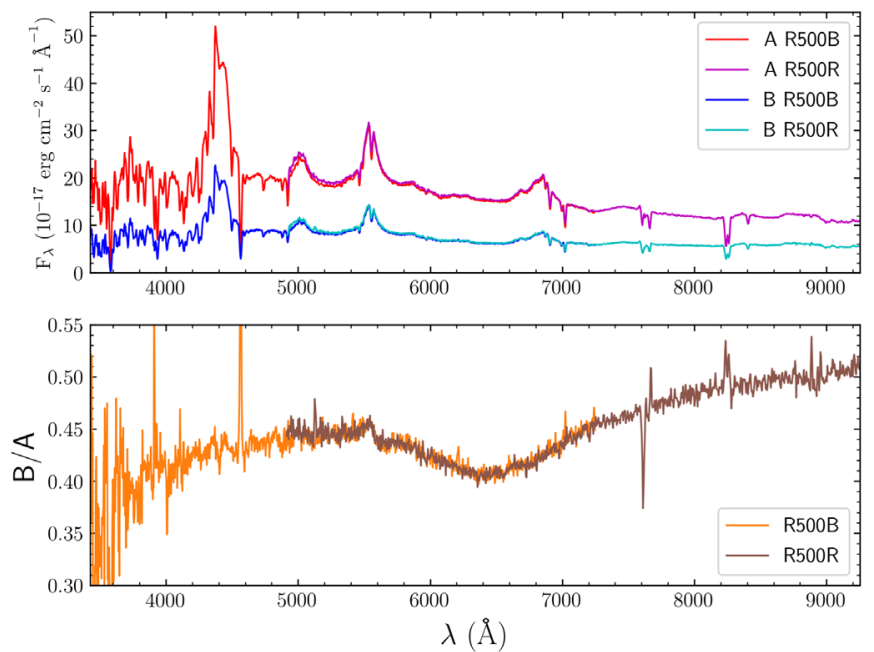

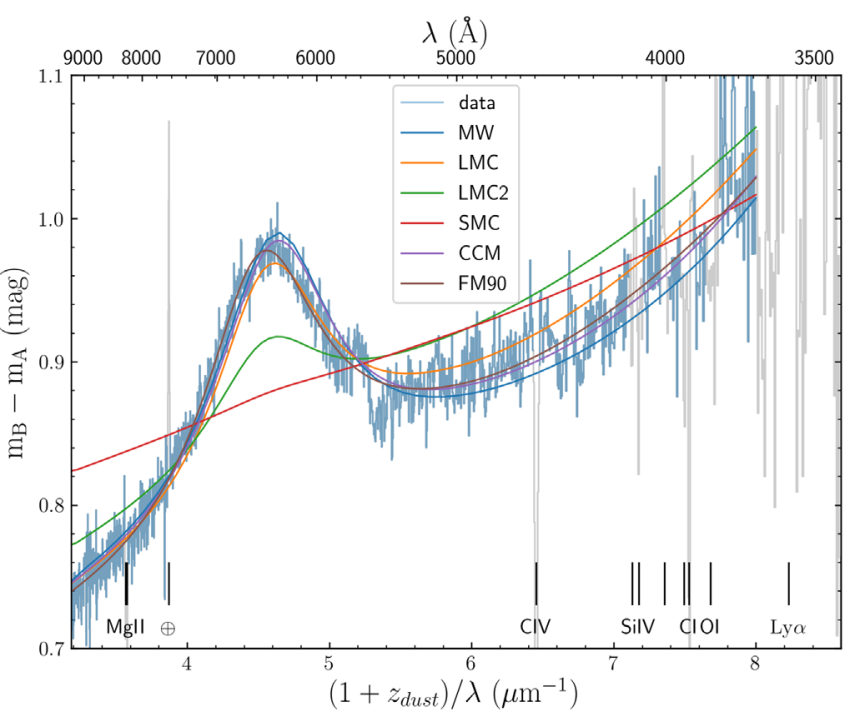

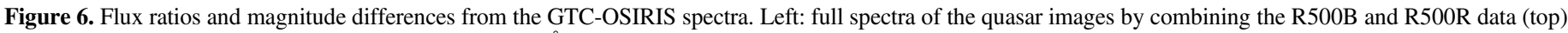

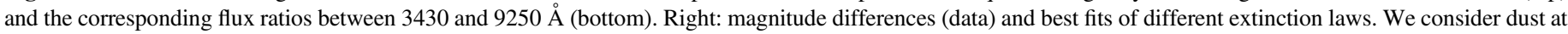

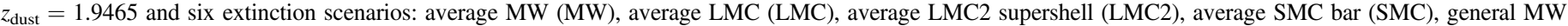

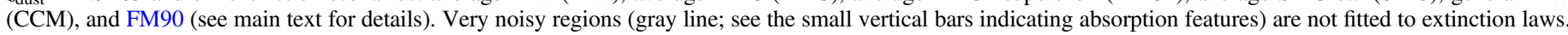

half those in $\mathrm{B}$, and noise is reduced when binning original data). In addition, selecting the method that produces the smallest uncertainty is a reasonable option (e.g., Tewes et al. 2013). Therefore, we adopted a delay interval of $25.0 \pm 1.5$ days (23.5-26.5 days).

Regarding the magnitude offsets, we derived the $1 \sigma$ intervals- $\Delta m_{\mathrm{AB}}(2016)=0.934 \pm 0.002 \mathrm{mag}, \Delta m_{\mathrm{AB}}(2017)=$ $0.956 \pm 0.002 \mathrm{mag}$, and $\Delta m_{\mathrm{AB}}(2018)=0.966 \pm 0.003 \mathrm{mag}-$ and we unambiguously detected microlensing-induced magnification gradients of $0.022 \pm 0.003 \mathrm{mag}$ (between 2016 and 2017) and $0.010 \pm 0.004 \mathrm{mag}$ (between 2017 and 2018). From the central values in the time delay and magnitude-offset intervals, we plotted the combined light curve in the $r$ band; i.e., the A brightness record and the magnitude- and time-shifted light curve of B are drawn together (see the bottom panel of Figure 5). We remark that by exclusively including seasonal changes in the $r$-band magnification ratio $\Delta m_{\mathrm{AB}}$, the shapes of $m_{\mathrm{A}}(t)$ and $m_{\mathrm{B}}\left(t+\Delta t_{\mathrm{AB}}\right)-\Delta m_{\mathrm{AB}}$ agree well with each other.

\section{Dust Extinction in a High-z, Galaxy}

For a double quasar, spectroscopic observations at two epochs separated by approximately the time delay between its two images lead to delay-corrected flux ratios at different wavelengths, which are valuable tools to study the macro- and microlens magnification ratios, as well as the differential dust extinction (e.g., Schneider et al. 2006). Our LT $r$-band monitoring of SDSS J1442+4055 yields a relatively short delay of about 25 days (A is leading; see Section 3), and we have checked that 25 days after the GTC-OSIRIS observations, the LT $r$-band flux of B only increased by $\sim 1 \%$. Additionally, the LT $r$-band flux of A at the GTC-OSIRIS observing epoch also increased by $\sim 1 \%$ compared to its value 25 days before (see Figure 2). Thus, considering calibration uncertainties and $e F_{\lambda} / F_{\lambda}$ values at red wavelengths, GTC-OSIRIS single-epoch flux ratios in the red spectral region seem to be plausible tracers of those corrected by intrinsic variability. Despite the spectra of SDSS J1442+4055AB being taken on a single night, we assumed that these data allow us to build flux ratios $B / A$
Table 5

Time Delay of SDSS J1442+4055

\begin{tabular}{lc}
\hline \hline Method & $\Delta t_{\mathrm{AB}}$ \\
\hline$\chi_{\mathrm{r}, \mathrm{A}}^{2}$ & $25.2_{-1.7}^{+1.1}$ \\
$\chi_{\mathrm{r}, \mathrm{B}}^{2}$ & $26.5 \pm 2.3$ \\
$D_{4,2}^{2}$ & $26.3_{-1.2}^{+3.5}$ \\
\hline
\end{tabular}

Note. Here $\Delta t_{\mathrm{AB}}$ is in days, $\mathrm{A}$ is leading, and all measurements are $68 \%$ confidence intervals.

describing the delay-corrected ones reasonably well. In the left panel of Figure 6, we present the single-epoch flux ratios from the GTC-OSIRIS spectra, which can be compared with the $A^{\star} / B^{\star}$ values in the top panel of Figure 2 of Krogager et al. (2018). Here contamination by light from G1 is denoted with a star superscript.

The $B / A$ data are very noisy at the shortest wavelengths, i.e., on the blue edge of the R500B grism (see the bottom subpanel in the left panel of Figure 6), which is partially due to the presence of a forest of absorption lines. Additional absorption features at longer wavelengths also produce spikes in $B / A$. However, in spectral intervals associated with broad lineemitting regions, no significant deviations are found with respect to adjacent continuum flux ratios. This suggests that chromatic microlensing is absent, since compact and extended emitting regions are magnified likewise. Therefore, we adopted a constant lens magnification ratio (including both a macrolens effect caused by the entire mass of the gravitational deflectors and a microlens effect produced by stars in intervening galaxies), so the chromatic behavior of $B / A$ is interpreted as due to dust extinction.

Apart from absorption-induced artifacts, the most dramatic feature in the flux ratio profile is a broad valley around $\lambda \sim$ $6400 \AA$, which is likely related to the $2175 \AA$ extinction bump seen in some galaxies of the Local Group (e.g., Gordon et al. 2003), lensing galaxies at $z \sim 1$ (e.g., Mediavilla et al. 2005), and several metal-rich absorbers at $z \sim 2$ (e.g., Ma et al. 2017). The 

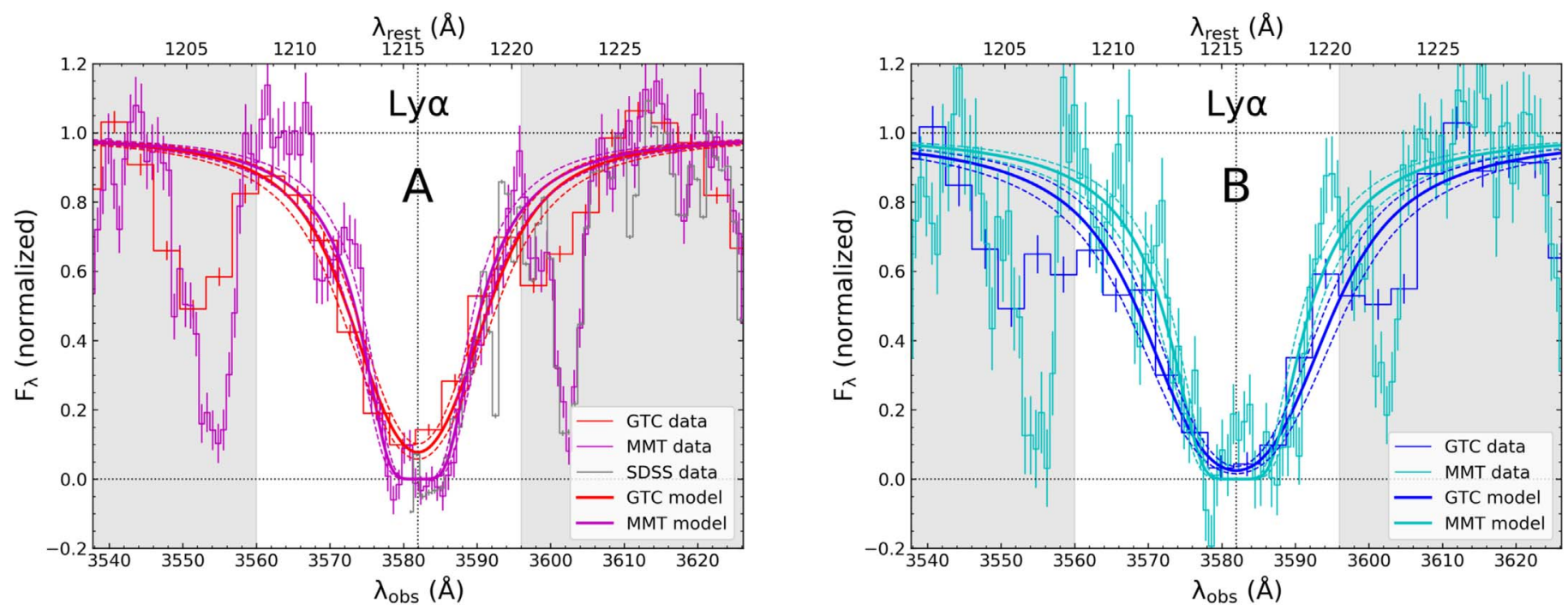

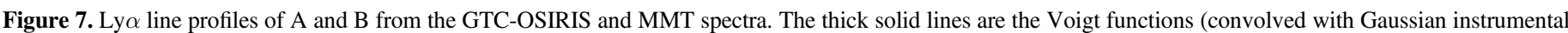

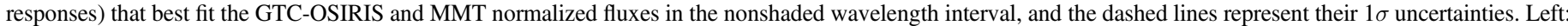

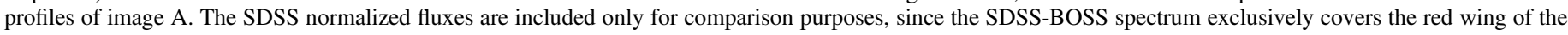
Ly $\alpha$ line. Right: profiles of image B.

existence of this bump is critical to deciding about the redshift of the intervening dust. Thus, we roughly obtained $z_{\text {dust }} \sim 1.94$, in good agreement with $z_{\text {IMS }}$. The main lensing galaxy G1 does not seem to play a relevant role in extinction, and the high-z IMS would be primarily responsible for the chromaticity observed in $B / A$. In fact, there is no appreciable absorption at $z_{\mathrm{G} 1}=0.284$ in the quasar spectra, while both spectra are clearly absorbed at $z_{\text {IMS }}=1.9465$. Figure 7 does not show any significant distortion of the Ly $\alpha$ profiles at $\lambda=3590 \AA$, where the $\mathrm{Mg}$ II 2796 line would have been seen if $\mathrm{Mg}$ II absorption had occurred in G1.

We converted flux ratios into magnitude differences, $m_{\mathrm{B}}-m_{\mathrm{A}}=-2.5 \log (B / A)$, and then used several extinction laws to fit these differences (e.g., Falco et al. 1999; Elíasdóttir et al. 2006). For $z_{\text {dust }}=1.9465$, we are probing the UV extinction in the distant dusty galaxy, i.e., at rest-frame wavelengths between 1165 and $3140 \AA$. This spectral range practically coincides with the wavelength coverage of the lowdispersion mode of the International Ultraviolet Explorer satellite, which provided a wealth of data on the UV extinction of close stars (e.g., Fitzpatrick \& Massa 1990). According to concordance cosmology with $H_{0}=70 \mathrm{~km} \mathrm{~s}^{-1} \mathrm{Mpc}^{-1}, \Omega_{m}=$ 0.27 , and $\Omega_{\Lambda}=0.73$ (Komatsu et al. 2009), the transverse distance between the two light paths at $z_{\text {dust }}$ (Smette et al. 1992; Cooke et al. 2010) is only $\sim 0.7 \mathrm{kpc}$ (see also Krogager et al. 2018). We implicitly assumed that the dust properties are the same along the lines of sight to both images. In addition, we did not fit the noisy magnitude differences at the shortest wavelengths or a series of spikes caused by absorption features at longer wavelengths (see the right panel of Figure 6).

First, we considered the average extinction curve of the Milky Way (MW; Cardelli et al. 1989), as well as average curves for other objects in the Local Group (Gordon et al. 2003): the Large Magellanic Cloud (LMC), LMC2 supershell (LMC2), and Small Magellanic Cloud bar (SMC). Our data are quite inconsistent with the average extinction curves of the LMC2 and SMC (green and red lines in the right panel of Figure 6) and show a behavior halfway between the average curves for the LMC and MW (orange and blue lines in the right panel of Figure 6). Second, we obtained a significant improvement in the reduced $\chi^{2}$ value when fitting a general Galactic extinction law (Cardelli et al. 1989, hereafter CCM). This CCM relationship led to a lens magnification ratio of $0.490 \pm 0.005 \mathrm{mag}$ (the constant term in $m_{\mathrm{B}}-m_{\mathrm{A}}$ ), a differential visual extinction $\Delta A_{\mathrm{AB}}(V)=A_{\mathrm{B}}(V)-A_{\mathrm{A}}(V)$ of $0.133 \pm 0.003 \mathrm{mag}$, and a total-to-selective extinction ratio $R$ $(V)$ of $2.672 \pm 0.048\left(\chi_{\mathrm{r}}^{2}=3.77\right.$; purple line in the right panel of Figure 6).

As a final step, in order to accurately describe the observed bump, data were fitted by the wavelength-dependent function of Fitzpatrick \& Massa (1990, hereafter FM90). FM90 introduced a Drude (Lorentzian-like) profile for representing a bump with central wavenumber $x_{0}=1 / \lambda_{0}$ and width (FWHM) $\gamma$, and $x_{0}=4.527 \pm 0.004 \mu \mathrm{m}^{-1}$ and $\gamma=0.99 \pm$ $0.02 \mu \mathrm{m}^{-1}$ were obtained from the fit $\left(\chi_{\mathrm{r}}^{2}=3.30\right.$; brown line in the right panel of Figure 6). In the right panel of Figure 6, the residuals of the purple and brown lines have amplitudes similar to those of the observed noise. Hence, although our best $\chi_{\mathrm{r}}^{2}$ values for the CCM and FM90 extinction laws are clearly greater than 1, formal uncertainties in magnitude differences may be underestimated by a factor $\sim 2$. While the value of $\gamma$ is typical for sight lines toward Galactic stars, the central wavelength of the extinction bump $\left(\lambda_{0}=2209 \pm 2 \AA\right)$ is extraordinarily unusual in the MW (e.g., Fitzpatrick \& Massa 2007). However, values of $x_{0}$ close to $4.53 \mu \mathrm{m}^{-1}$ are consistent with measurements in the LMC (e.g., Gordon et al. 2003) and some metal-rich absorbers at $z \sim 1-2$ (e.g., Ma et al. 2017).

\section{High- $z$ Gas and Its Correlation with Dust}

\subsection{Complementary Observations}

To analyze the gas content of the IMS is of interest not only in the use of the low-resolution GTC-OSIRIS spectra of both quasar images (resolving power of $~ 300-400$; see Section 2.2) but also other available, not previously analyzed mediumresolution spectroscopic data. This higher resolution allows us to identify finer spectral details, e.g., resolve blended 
Table 6

Neutral-hydrogen Column Densities

\begin{tabular}{lcc}
\hline \hline Facility & $\log N_{\mathrm{A}}(\mathrm{H} \mathrm{I})^{\mathrm{a}}$ & $\log N_{\mathrm{B}}(\mathrm{H} \mathrm{I})^{\mathrm{a}}$ \\
\hline GTC-OSIRIS & $20.160 \pm 0.021$ & $20.490 \pm 0.029$ \\
MMT & $20.130 \pm 0.015$ & $20.279 \pm 0.025$ \\
\hline
\end{tabular}

Note.

${ }^{\text {a }}$ Column density $N$ in $\mathrm{cm}^{-2}$.

absorption lines. Therefore, in addition to the GTC-OSIRIS data, we used the SDSS-BOSS spectrum of the A image with a resolving power of $\sim 2000$, as well as the data that were obtained at the MMT Observatory with the Blue Channel Spectrograph (Findlay et al. 2018). The MMT spectra of A and B on 2015 June 14 cover a wavelength range of 3500-5500 at a spectral resolution of $\sim 1800$, which is about 5 times higher than those of the R500B and R500R grisms. For each spectrum, we fitted a global continuum and obtained normalized fluxes using the Linetools software. ${ }^{8}$

\subsection{Neutral Hydrogen}

The GTC-OSIRIS and MMT spectra cover the Ly $\alpha$ absorption at $z_{\text {gas }}=1.9465$, which is observed around $3582 \AA$. Regarding the GTC-OSIRIS spectra, the Ly $\alpha$ line profile of the $\mathrm{B}$ image is deeper and wider than that of the $\mathrm{A}$ image, and this suggests a larger $\mathrm{H} \mathrm{I}$ column density along the line of sight to B. Using both data sets at different spectral resolutions, we fitted line profiles to a Voigt function convolved with a Gaussian instrumental profile (Krogager 2018). These fits were performed with the VoigtFit software. ${ }^{9}$ In Figure 7, we show the best fits (thick solid lines) along with their $1 \sigma$ uncertainties (dashed lines). We note that the fits were done by minimizing $\chi^{2}$ in the interval $3560 \AA \leqslant \lambda_{\text {obs }} \leqslant$ $3596 \AA$ (central, nonshaded region in the two panels of Figure 7), so that we avoided the Si III 1206 line and another prominent absorption feature at $\lambda_{\text {obs }}=3603 \AA$. Furthermore, in order to estimate $1 \sigma$ confidence intervals, we used 1000 repetitions of each $\operatorname{Ly} \alpha$ profile. To obtain a repetition of an original Ly $\alpha$ profile, we modified the normalized observed fluxes by adding realizations of normal distributions around zero, with standard deviations equal to the measured errors.

The GTC-OSIRIS and MMT data yield the neutral-hydrogen column densities in Table 6. It is evident that both measures of $\log N_{\mathrm{B}}(\mathrm{H} \mathrm{I})$ differ by $\sim 0.2$, which is an order of magnitude larger than the formal errors. Thus, we adopted a statistical approach, considering the two values in Table 6 (20.490 and 20.279) and $\log N_{\mathrm{B}}(\mathrm{H} \mathrm{I})$ from the rest-frame equivalent width (EW) of the Ly $\alpha$ line in the MMT spectrum of B (20.26; see Equation (9.24) of Draine 2011). Calculating the average value and its standard deviation, and taking into account that the standard deviation of the mean of three values is $50 \%$ uncertain, we obtain $\log N_{\mathrm{B}}(\mathrm{HI})=20.34 \pm 0.11$. From the two values of $\log N_{\mathrm{A}}(\mathrm{HI})$ in Table 6 , we also infer $\log N_{\mathrm{A}}(\mathrm{H} \mathrm{I})=20.14 \pm 0.11$, where the error of the mean was conservatively enlarged to 0.11 . The IMS can be classified as a

\footnotetext{
8 Linetools is a Python package mainly aimed at the identification and analysis of absorption lines in quasar spectra. This is publicly available at https://github.com/profxj/linetools.

9 VoigtFit is a Python package for Voigt profile fitting that is publicly available at https://github.com/jkrogager/VoigtFit.
}

subdamped/damped Ly $\alpha$ (subDLA/DLA) system (Wolfe et al. 1986), and our H I column densities agree (although having larger uncertainties) with those from the high-resolution KeckHIRES spectra of the quasar at $\lambda<6000 \AA$ on 2017 May 20 (Krogager et al. 2018).

\subsection{Dust-to-gas Ratio}

The visual extinction $A(V)$ is proportional to the optical depth at $\lambda_{\text {rest }}=0.55 \mu \mathrm{m}$, which in turn is proportional to the dust grain column density $N$ (dust). Assuming that $N$ (dust) $\propto N(\mathrm{HI})$ (e.g., Fall \& Pei 1989; Zuo et al. 1997), we then obtained $A_{\mathrm{B}}(V) / A_{\mathrm{A}}(V)=N_{\mathrm{B}}(\mathrm{H} \mathrm{I}) / N_{\mathrm{A}}(\mathrm{H} \mathrm{I}) \sim 1.6$ (see Section 5.2). From this visual extinction ratio and the differential visual extinction in Section 4, it is possible to estimate the effect of dust along each line of sight: $A_{\mathrm{A}}(V) \sim 0.22$ and $A_{\mathrm{B}}(V) \sim 0.35 \mathrm{mag}$. We remark upon the similarity between these individual extinctions and the $A(V)$ values found in Krogager et al. (2018). The color excesses of the individual images would be $E_{\mathrm{A}}(B-V) \sim 0.08$ and $E_{\mathrm{B}}(B-V) \sim 0.13 \mathrm{mag}$; thus, the bump strength (area of the extinction bump) may be estimated at 0.47 and $0.76 \mathrm{mag} \mu \mathrm{m}^{-1}$ for $\mathrm{A}$ and $\mathrm{B}$, respectively. These strengths agree well with those of the LMC and metal-rich absorbers at $z \sim 1-2$, whereas they are weaker than most measures in the MW (see Figure 1 of Ma et al. 2017).

The ratio between $A(V)$ (or $E(B-V)$ ) and $N(\mathrm{H} \mathrm{I})$ is usually called the dust-to-gas ratio (e.g., Ma et al. 2018 and references therein). For the IMS of SDSS J1442+4055, we derived $A(V) / N(\mathrm{H} \mathrm{I}) \sim 1.6 \times 10^{-21} \mathrm{mag} \mathrm{\textrm {cm } ^ { 2 }}$, and such a high value is also observed in some absorbers with high metallicity (see Section 5.5). This dust-to-gas ratio is a factor of $\sim 3$ higher than that of the local interstellar medium (Liszt 2014) and the MW average visual extinction per $\mathrm{H}$ for $R(V) \sim 2.7$ (see Figure 3 of Draine 2003), as well as about 5 times higher than the mean ratio of the LMC and $\mathrm{Mg}$ II absorbers (Gordon et al. 2003; Ménard \& Chelouche 2009). Moreover, the mean ratio of high- $z$ DLAs is nearly two orders of magnitude lower than the $A(V) / N$ (H I) value for the IMS (Vladilo et al. 2008). Lastly, it is worth mentioning that $E(B-V) / N(\mathrm{HI}) \sim 6 \times 10^{-22}$ mag cm${ }^{2}$, which is similar to the corresponding ratio of the distant lensing galaxy of SBS 0909+532 (Dai \& Kochanek 2009).

\subsection{Metals}

In addition to neutral hydrogen, quasar spectra are affected by metals at $z_{\text {gas }}=1.9465$. Prominent metal lines are shown in Figure 8, which incorporates data from the SDSS-BOSS (gray; only for A), GTC-OSIRIS (red for A and blue for B), and MMT Observatory (magenta for A and cyan for B). The GTCOSIRIS line profiles were then used to determine the rest-frame EWs of absorption lines at $\lambda_{\text {obs }} \geqslant 5500 \AA$, whereas the EWs of lines observed at shorter wavelengths were derived from the MMT profiles (see Section 5.1). The EWs of the main absorption features are listed in Table 7. The neutral-carbon detection is particularly interesting, since the appearance of a $2175 \AA$ extinction bump at $z \sim 2$ has been linked to the presence of C I (e.g., Elíasdóttir et al. 2009; Ledoux et al. 2015; Ma et al. 2018). We observe strong absorption (EW $>1 \AA$ ) of C I 1328 and weaker lines of C I 1277, C I 1560, and C I 1656. We also note the presence of Zn II 2026. Despite the Zn II 2026 and $\mathrm{Mg}$ I 2026 lines are blended with each other, EW(Zn II 2026) values are presented in Table 7 . We used the correction 


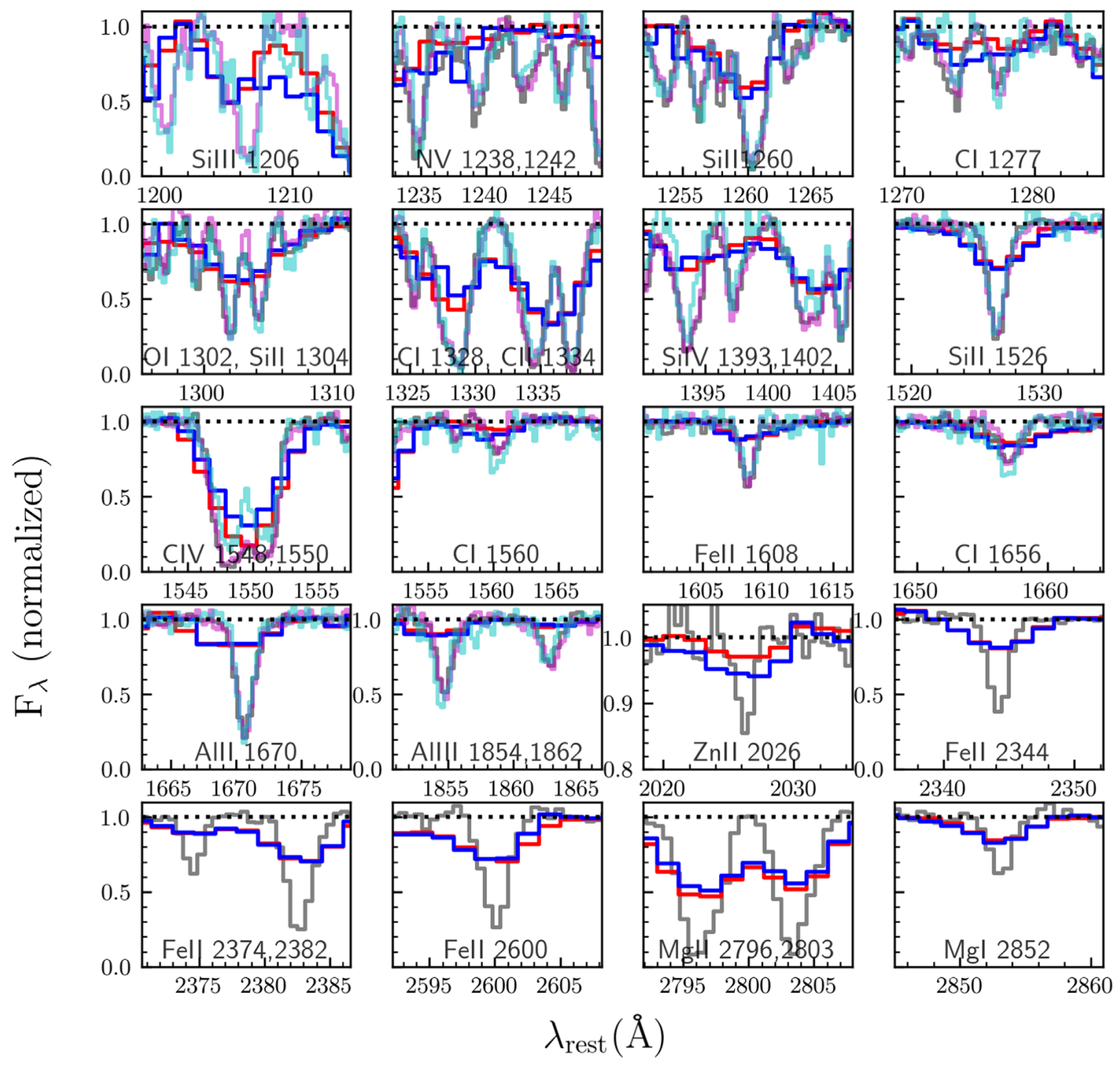

Figure 8. Prominent metal absorption lines due to the IMS toward SDSS J1442+4055. Profiles of A are marked in gray (SDSS-BOSS data), red (GTC-OSIRIS data), and magenta (MMT data), and profiles of B are marked in blue (GTC-OSIRIS data) and cyan (MMT data).

$\mathrm{EW}(\mathrm{Zn}$ II 2026) $=\mathrm{EW}(\mathrm{Zn}$ II $2026+\mathrm{Mg} \mathrm{I} 2026)-[f(\mathrm{Mg} \mathrm{I}$ 2026) $/ f(\operatorname{Mg}$ I 2852) $] \times \mathrm{EW}\left(\mathrm{Mg}_{\mathrm{I}} 2852\right)$, where $f(\operatorname{Mg}$ I 2026) and $f(\mathrm{Mg} \mathrm{I} 2852)$ are oscillator strengths.

As a first approximation, $\mathrm{EW}_{\mathrm{B}} / \mathrm{EW}_{\mathrm{A}}$ ratios were used to estimate relative amounts of neutral hydrogen and metals along the two sight lines (see Figure 9). These quantities approximately represent column density ratios $N_{\mathrm{B}} / N_{\mathrm{A}}$ at low optical depths, but they describe $\left(N_{\mathrm{B}} / N_{\mathrm{A}}\right)^{1 / 2}$ values at very high optical depths (e.g., Draine 2011). Thus, in Figure 9, we have to remark that $\mathrm{EW}_{\mathrm{B}}(\mathrm{HI}) / \mathrm{EW}_{\mathrm{A}}(\mathrm{HI}) \sim\left[N_{\mathrm{B}}(\mathrm{H} \mathrm{I}) / N_{\mathrm{A}}(\mathrm{H} \mathrm{I})\right]^{1 / 2}$. Section 5.2 provides data for a reliable estimation of $N_{\mathrm{B}}(\mathrm{H} \mathrm{I}) /$ $N_{\mathrm{A}}(\mathrm{HI})$. In Figure 9, we differentiate between neutral atoms (blue circles), low-ionization metals (green circles), and highionization metals (red circles).

The C I 1560 and 1656 absorption lines (see Table 7) are formed at relatively low optical depths, so their EW ratios roughly correspond to column density ratios. We thus obtain that the neutral-carbon column density for B (the most reddened image) is appreciably higher than that for A. From the Keck-HIRES high-resolution spectra, Krogager et al. (2018) also found an $N_{\mathrm{B}}(\mathrm{CI}) / N_{\mathrm{A}}(\mathrm{CI})$ ratio above 3. Even though both images seem to be affected by similar amounts of Fe II and $\mathrm{Mg}$ II, the $\mathrm{Zn}$ II absorption is stronger along the line of sight to B. Nonrefractory (volatile) elements, e.g., Zn, condense onto dust grains much more difficultly than refractory elements, e.g., Fe and $\mathrm{Mg}$. Hence, in the $\mathrm{B}$ image, we observe a relative excess of $\mathrm{Zn}$ in the gas phase. However, there are no gas-phase excesses of $\mathrm{Fe}$ and $\mathrm{Mg}$, which are more easily trapped in dust grains. It is also clear that the A image is more affected by high-ionization metals.

\subsection{Metallicity and Dust Depletion}

The two absorption lines of Fe II 1608 and Zn II 2026 are not saturated and lie outside the Ly $\alpha$ forest. Thus, we initially used Equation (9.15) of Draine (2011) to estimate column densities from their EWs. However, if the column densities are not actually proportional to the EWs (the optically thin regime condition is not met), the $N$ values are underestimated, being underestimated greater when the EW is larger. While the EWs of the $\mathrm{Zn}$ II 2026 line do not exceed $0.3 \AA$, the EWs of the Fe II 1608 line reach $0.6 \AA$, and we carried out a more detailed analysis of this stronger absorption. For the A image, the optically 


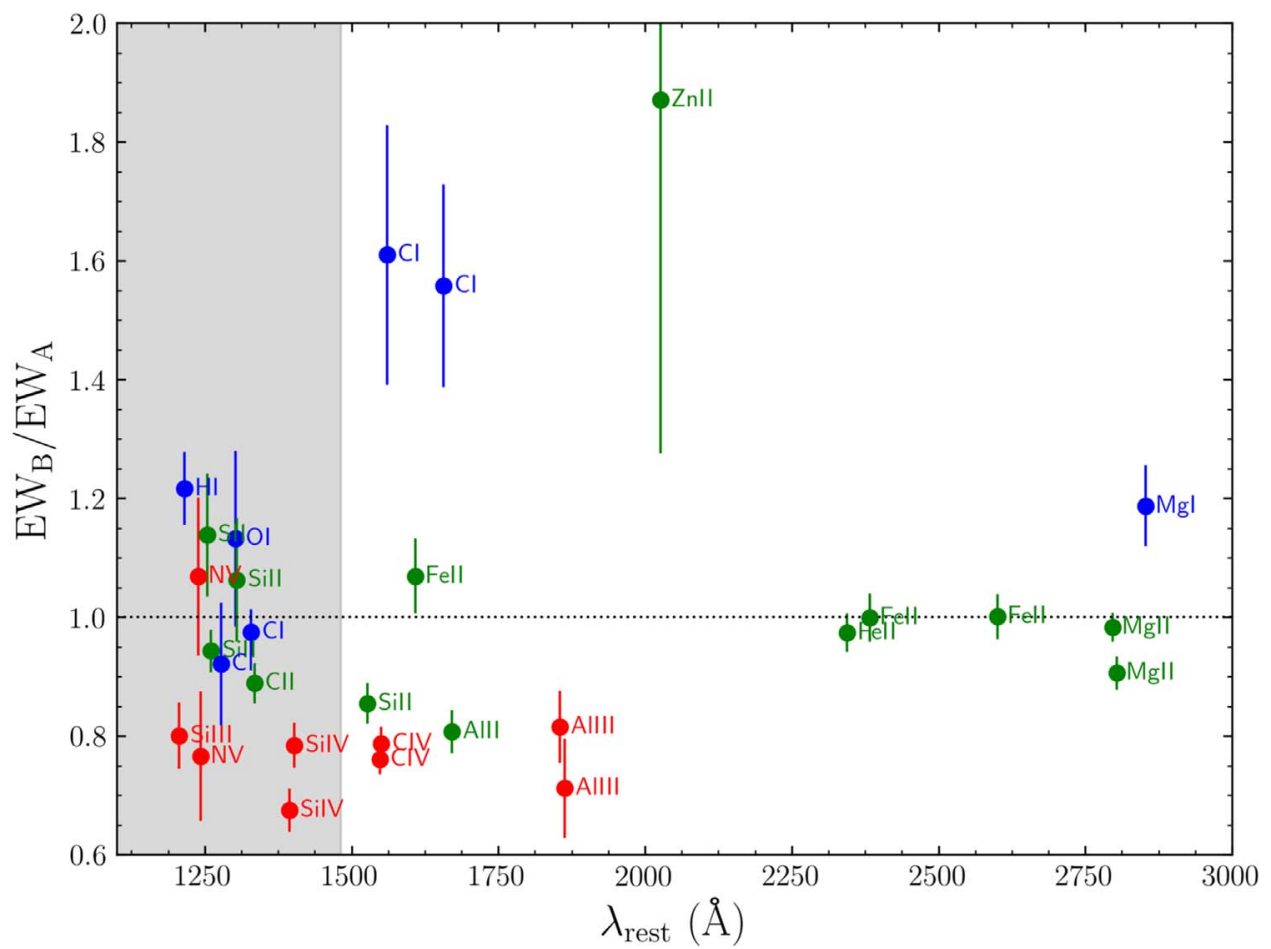

Figure 9. EW ratios. We highlight absorption features blueward of the Ly $\alpha$ emission of the quasar (gray region), because some of them could be blended with lines belonging to the $\mathrm{Ly} \alpha$ forest. Blue, green, and red circles denote ratios of neutral atoms, low-ionization metals, and high-ionization metals, respectively.

Table 7

Rest-frame EWs of the Main Absorption Lines Produced by the IMS

\begin{tabular}{lcccc}
\hline \hline Ion & $\lambda_{\text {rest }}{ }^{\mathrm{a}}$ & $\mathrm{EW}_{\mathrm{A}}{ }^{\mathrm{b}}$ & $\mathrm{EW}_{\mathrm{B}}{ }^{\mathrm{b}}$ & Data \\
\hline $\mathrm{Si}$ III & 1206.50 & $2.183 \pm 0.080$ & $1.747 \pm 0.105$ & MMT \\
$\mathrm{H} \mathrm{I}$ & 1215.67 & $8.137 \pm 0.215$ & $9.899 \pm 0.420$ & MMT \\
$\mathrm{N} \mathrm{V}$ & 1238.82 & $0.777 \pm 0.053$ & $0.830 \pm 0.086$ & MMT \\
$\mathrm{N} \mathrm{V}$ & 1242.80 & $0.768 \pm 0.046$ & $0.588 \pm 0.076$ & MMT \\
$\mathrm{S}$ II & 1253.80 & $0.767 \pm 0.040$ & $0.873 \pm 0.065$ & MMT \\
$\mathrm{Si} \mathrm{II}$ & 1260.42 & $1.910 \pm 0.037$ & $1.801 \pm 0.058$ & MMT \\
$\mathrm{C}$ I & 1277.24 & $0.723 \pm 0.048$ & $0.666 \pm 0.060$ & MMT \\
O I & 1302.17 & $0.963 \pm 0.040$ & $1.090 \pm 0.135$ & MMT \\
Si II & 1304.37 & $0.767 \pm 0.035$ & $0.815 \pm 0.071$ & MMT \\
C I & 1328.83 & $2.622 \pm 0.052$ & $2.555 \pm 0.089$ & MMT \\
C II & 1334.53 & $2.516 \pm 0.048$ & $2.236 \pm 0.074$ & MMT \\
Si IV & 1393.78 & $1.880 \pm 0.043$ & $1.269 \pm 0.062$ & MMT \\
Si IV & 1402.77 & $1.856 \pm 0.040$ & $1.455 \pm 0.063$ & MMT \\
Si II & 1526.70 & $1.286 \pm 0.034$ & $1.099 \pm 0.034$ & MMT \\
C IV & 1548.20 & $2.627 \pm 0.040$ & $1.998 \pm 0.058$ & MMT \\
C IV & 1550.77 & $2.238 \pm 0.039$ & $1.761 \pm 0.055$ & MMT \\
C I & 1560.30 & $0.287 \pm 0.031$ & $0.462 \pm 0.038$ & MMT \\
Fe II & 1608.45 & $0.565 \pm 0.018$ & $0.604 \pm 0.030$ & MMT \\
C I & 1656.92 & $0.443 \pm 0.037$ & $0.690 \pm 0.049$ & MMT \\
Al II & 1670.78 & $1.391 \pm 0.041$ & $1.123 \pm 0.038$ & MMT \\
Al III & 1854.71 & $0.937 \pm 0.031$ & $0.764 \pm 0.051$ & MMT \\
Al III & 1862.78 & $0.587 \pm 0.030$ & $0.418 \pm 0.044$ & MMT \\
Zn II & 2026.13 & $0.170 \pm 0.038$ & $0.318 \pm 0.072$ & GTC-OSIRIS \\
Fe II & 2344.21 & $1.527 \pm 0.025$ & $1.487 \pm 0.043$ & GTC-OSIRIS \\
Fe II & 2382.76 & $1.614 \pm 0.032$ & $1.612 \pm 0.057$ & GTC-OSIRIS \\
Fe II & 2600.17 & $1.824 \pm 0.032$ & $1.825 \pm 0.062$ & GTC-OSIRIS \\
Mg II & 2796.35 & $3.625 \pm 0.045$ & $3.563 \pm 0.077$ & GTC-OSIRIS \\
Mg II & 2803.53 & $2.417 \pm 0.035$ & $2.189 \pm 0.060$ & GTC-OSIRIS \\
Mg I & 2852.96 & $0.781 \pm 0.024$ & $0.927 \pm 0.045$ & GTC-OSIRIS \\
\hline
\end{tabular}

Notes.

${ }^{a}$ Central rest-frame wavelength in $\AA$.

${ }^{\mathrm{b}}$ Rest-frame EW in $\AA$.
Table 8

Fe II and Zn II Column Densities

\begin{tabular}{lcc}
\hline \hline Ion & $\log N_{\mathrm{A}}{ }^{\mathrm{a}}$ & $\log {N_{\mathrm{B}}}^{\mathrm{a}}$ \\
\hline Fe II & $14.71 \pm 0.04$ & $14.74 \pm 0.04$ \\
Zn II & $12.97 \pm 0.10$ & $13.24 \pm 0.10$ \\
\hline
\end{tabular}

Note.

${ }^{\text {a }}$ Column density $N$ in $\mathrm{cm}^{-2}$.

thin regime approach led to $\log N_{\mathrm{A}}$ (Fe II $)=14.63 \pm 0.01$. Additionally, the SDSS-BOSS spectrum of A allowed us to construct the curve of growth for Fe II, as well as to fit the two relevant parameters: $\log N_{\mathrm{A}}(\mathrm{Fe}$ II $)=14.71 \pm 0.04$ and $b=69 \pm$ $3 \mathrm{~km} \mathrm{~s}^{-1}$. We adopted this last interval for $\log N_{\mathrm{A}}(\mathrm{Fe}$ II $)$ and considered a bias of -0.08 to correct our initial estimate of $\log N_{\mathrm{B}}$ (Fe II) through the optically thin regime approach (its error was also set to 0.04 ; see Table 8 ).

In Table 9, we also present the metal abundances $[\mathrm{Fe} / \mathrm{H}]$ and $[\mathrm{Zn} / \mathrm{H}]$, where $[\mathrm{X} / \mathrm{H}]=\log [N(\mathrm{X}) / N(\mathrm{H})]-\log (\mathrm{X} / \mathrm{H})_{\odot} . \mathrm{We}$ assumed that $N(\mathrm{Fe})=N(\mathrm{Fe}$ II $), N(\mathrm{Zn})=N(\mathrm{Zn} \mathrm{II})$, and $N$ $(\mathrm{H})=N(\mathrm{HI})$ and took the solar abundances $\log (\mathrm{X} / \mathrm{H})_{\odot}$ from Asplund et al. (2009). Using the average value of $[\mathrm{Zn} / \mathrm{H}]$ as a metallicity estimator ( $\mathrm{Zn}$ basically remains in the gas phase), the IMS has a supersolar metallicity $[\mathrm{Zn} / \mathrm{H}]_{\mathrm{IMS}}=+0.27$. This means that $(\mathrm{Zn} / \mathrm{H})_{\text {IMS }}$ is about 2 times $(\mathrm{Zn} / \mathrm{H})_{\odot}$. However, we should bear in mind that Krogager et al. (2018) detected $\mathrm{H}_{2}$ along both lines of sight, so that when using the total hydrogen column density instead of $N(\mathrm{HI}), \log N(\mathrm{H})$ must be increased by about $1 \%$. As a result, $\left[\mathrm{Zn} / \mathrm{H}_{\mathrm{T}}\right]_{\mathrm{IMS}} \sim+0.07$, which confirms the metallicity from sulfur and total hydrogen $\left[\mathrm{S} / \mathrm{H}_{\mathrm{T}}\right]_{\mathrm{IMS}} \sim 0$, based on high-resolution Keck-HIRES spectra. In addition, the $[\mathrm{Fe} / \mathrm{H}]$ values are significantly less than zero, indicating depletion 
Table 9

Fe and $\mathrm{Zn}$ Abundances Relative to Their Solar Values

\begin{tabular}{|c|c|c|c|c|}
\hline & \multicolumn{2}{|c|}{$[\overline{[\mathrm{Fe} / \mathrm{H}]}$} & \multicolumn{2}{|c|}{ [ZZ/H] } \\
\hline & $\mathrm{A}$ & B & A & B \\
\hline Photosphere & $-0.93 \pm 0.12$ & $-1.10 \pm 0.12$ & $+0.27 \pm 0.16$ & $+0.34 \pm 0.16$ \\
\hline Meteorites & $-0.88 \pm 0.12$ & $-1.05 \pm 0.12$ & $+0.20 \pm 0.15$ & $+0.27 \pm 0.15$ \\
\hline
\end{tabular}

Note. We consider solar photosphere and meteorite metal abundances in Table 1 of Asplund et al. (2009).

of refractory elements onto dust grains. The abundance ratio of iron to zinc, $[\mathrm{Fe} / \mathrm{Zn}]$, is commonly used as a dust depletion estimator. It measures the depletion of Fe from its gas phase to the dust phase. The column densities in Table 8 and solar metal abundances in Table 1 of Asplund et al. (2009) yielded $[\mathrm{Fe} / \mathrm{Zn}]_{\mathrm{A}}=-1.14 \pm 0.11$ and $[\mathrm{Fe} / \mathrm{Zn}]_{\mathrm{B}}=-1.38 \pm 0.11$, where $\log (\mathrm{Fe} / \mathrm{Zn})_{\odot}$ was averaged over its photosphere and meteorite values.

The IMS of SDSS J1442+4055 belongs to the family of $2175 \AA$ dust absorbers (2DAs) that was studied by Ma et al. (2018), who also assumed $N(\mathrm{H})=N(\mathrm{HI})$. The 2DAs contain C I absorbing gas with $\log N(\mathrm{C}$ I $)>14.0$ (we obtain $\log N(\mathrm{C}$ I) $>14.0$ from the EWs of the C I 1656 line in Table 7), and the subDLAs/DLAs with $\log N(\mathrm{HI}) \sim 20.0-20.5$ (subset of the 2DA population) have supersolar metallicities (see Table 9). These 2DAs also show a strong correlation between dust-to-gas ratio and metallicity. For the dust-to-gas ratio of the IMS (see Section 5.3), Equation (7) of Ma et al. (2018) predicts a high metallicity, $[\mathrm{Zn} / \mathrm{H}] \sim+0.3$, in good agreement with the values in Table 9. In addition, if we focus on the 2DAs with $\log N(\mathrm{H} \mathrm{I}) \sim 20.0-20.5$, their high depletion levels agree well with our measures of $[\mathrm{Fe} / \mathrm{Zn}]$ (see above). The values of $[\mathrm{Fe} / \mathrm{Zn}]_{\mathrm{A}}$ and $[\mathrm{Fe} / \mathrm{Zn}]_{\mathrm{B}}$ can be used to estimate the stellar mass of the IMS. From the mass-metallicity-redshift relation of Møller et al. (2013) and the observed $[\mathrm{Zn} / \mathrm{H}]-[\mathrm{Fe} / \mathrm{Zn}]$ relationship in the 2DAs, we derived $M_{\text {stars }} \sim 2 \times 10^{10} M_{\odot}$. Therefore, the IMS host galaxy appears to be a metal-rich and relatively massive object containing large amounts of dust and neutral gas. In this scenario, star formation is likely to occur.

\section{Conclusions}

This paper mainly reports on optical follow-up observations of the gravitationally lensed quasar SDSS J1442+4055 using the GTC and LT. The main lensing galaxy, G1, is only 1!"38 from the brightest quasar image, A, and its GTC spectra clearly show the $\mathrm{Ca}$ II $\mathrm{HK}, G$-band, $\mathrm{H} \beta$, and $\mathrm{Mg}$ I $b$ absorption features at $z_{\mathrm{G} 1}=0.284 \pm 0.001$. The new spectra of $\mathrm{G} 1$ with unprecedented quality might be used (together with IR spectroscopy) to fit stellar population models in the nonlocal early-type galaxy (e.g., Bruzual 2003). This should lead to realistic microlens mass functions to generate microlensing magnification maps. The secondary galaxies G2 and G3 are located 5!" 2 and 33!" 9 from A, and our LT spectra of these two objects yield redshifts $z_{\mathrm{G} 2}=z_{\mathrm{G} 3}=0.22 \pm 0.01$. Thus, $\mathrm{G} 2$ is not physically associated with $\mathrm{G} 1$, but it is at the same distance as G3.

The LT $r$-band light curves of the two quasar images A and B over $2.7 \mathrm{yr}$ of monitoring display significant variations, which are used to measure a time delay of $25.0 \pm 1.5$ days $(1 \sigma$ confidence interval; $\mathrm{A}$ is leading). Despite this delay being robustly measured to $6 \%$ precision, before using it to estimate cosmological parameters, one must consider a possible microlensing-induced contribution (Tie \& Kochanek 2018). To properly account for a putative microlensing bias in the time-delay estimation, it is required to perform numerical simulations. However, there are reasons to think this bias is well below the delay uncertainty of 1.5 days. First, we detect microlensing magnification gradients $<10^{-4}$ mag day $^{-1}$ in the $r$ band. Second, the flux ratios $B / A$ from the GTC spectra of both quasar images do not show evidence of microlensing inhomogeneous magnification, since sources with different shapes/sizes are magnified equally.

Current observational constraints also allow us to explore simple mass models for SDSS J1442+4055 and thus compare the predicted delays with the measured one. We may consider the astrometry in Table 1 of Sergeyev et al. (2016) and the lens magnification ratio we derive in Section 4, i.e., a macrolens flux ratio $B / A=0.64 \pm 0.064$, where the uncertainty is increased to $10 \%$ to take an unknown microlens effect into account. If we fit a singular isothermal ellipsoid (SIE) mass model to these observations $\left(\chi^{2} \sim 0\right)$, the LENSMODEL software (Keeton 2001, 2010) produces an Einstein radius, ellipticity (position angle), and time delay of $1 . " 073,0.034$ $\left(-28^{\circ} .0\right)$, and 26 days (adopting the concordance cosmology we use in Section 4; Komatsu et al. 2009). As the ellipticity of the SIE model is quite small, we could also probe a singular isothermal sphere (SIS), where the position of the SIS is allowed to vary during the fitting procedure. Through the LENSMODEL package, we find a solution with $\chi_{\mathrm{r}}^{2}=$ $\chi^{2} /$ dof $=2.45 / 2$. The lensing mass parameters are 1 !" 078 (Einstein radius) and $\left(x_{\text {lens }}, y_{\text {lens }}\right)=(1$."339, -0 ". 323$)$, with the lens center being slightly offset $(\sim 0$ " 02$)$ from Sergeyev et al.'s position of G1. This SIS model leads to a time delay of 25.3 days, which is very close to our central delay value (see above).

The GTC quasar spectra indicate the presence of an IMS at $z \sim 2$, and we measure $z_{\mathrm{IMS}}=1.9465$ from strong metal absorption lines in the SDSS-BOSS spectrum of A, which has a higher resolution than those of the GTC (see also Sergeyev et al. 2016; Krogager et al. 2018). Leaving aside absorption features, the high-S/N spectroscopy with the GTC offers a unique opportunity to analyze the flux ratios $B / A$ over the wide wavelength interval between 3430 and $9250 \AA$. A prominent extinction bump is detected at a redshift similar to that of the distant IMS, so this high- $z$ object contains dust grains and gasphase metals. Assuming that dust properties are similar along both sight lines (A and B), we fit extinction curves to the magnitude differences from the measured flux ratios. At $z_{\mathrm{IMS}}=1.9465$, the transverse distance between A and B is less than $1 \mathrm{kpc}$. In addition, Østman et al. (2008) reported that when several images of the same quasar are affected by dust extinction, the preferred values of $R(V)$ are similar. A general Galactic extinction curve (Cardelli et al. 1989) yields an acceptable fit with $R(V)=2.672 \pm 0.048$ and a differential visual extinction $A_{\mathrm{B}}(V)-A_{\mathrm{A}}(V)=0.133 \pm 0.003$ mag. Moreover, using the extinction law of Fitzpatrick \& Massa (1990), we 
obtain $x_{0}=4.527 \pm 0.004 \mu \mathrm{m}^{-1}$ and $\gamma=0.99 \pm 0.02 \mu \mathrm{m}^{-1}$ for the central wavenumber and width (FWHM) of the extinction bump. The value of $x_{0}$ is very unusual in the MW, but it agrees with the values in the LMC and metal-rich absorbers at $z \sim 1-2$ (e.g., Gordon et al. 2003; Fitzpatrick \& Massa 2007; Ma et al. 2017).

To accurately study the gas content of the high- $z$ IMS and the dust-gas correlation, we use the GTC spectra of A and B, as well as higher-resolution data from the SDSS-BOSS spectroscopy of A and the MMT observations of both quasar images (Findlay et al. 2018). Assuming that the dust grain column density is proportional to the $\mathrm{HI}$ column density, the visual extinction ratio $A_{\mathrm{B}}(V) / A_{\mathrm{A}}(V)=N_{\mathrm{B}}(\mathrm{H} \mathrm{I}) / N_{\mathrm{A}}(\mathrm{H} \mathrm{I}) \sim 1.6$ enables us to know how dust affects each individual image. For example, we estimate bump strengths of 0.47 (A) and 0.76 (B) $\mathrm{mag} \mu \mathrm{m}^{-1}$. These are consistent with bump areas in the LMC and metal-rich absorbers at $z \sim 1-2$ (e.g., Ma et al. 2017). The IMS at $z \sim 2$ belongs to the family of dusty absorbers discussed by Ma et al. (2018), since it is a metal-strong subDLA/DLA system with $\log N(\mathrm{HI}) \sim 20.0-20.5$, contains $\mathrm{CI}$ gas with $\log N(\mathrm{C} \mathrm{I})>$ 14.0, and has high values of the dust-to-gas ratio $A(V) / N(\mathrm{HI})$ $\left.\left(\sim 1.6 \times 10^{-21} \mathrm{mag} \mathrm{cm}\right)^{2}\right)$, the gas-phase metallicity indicator $[\mathrm{Zn} / \mathrm{H}](\sim+0.3 ; \mathrm{H} \equiv \mathrm{HI})$, and the dust depletion level $(-1.5<[\mathrm{Fe} / \mathrm{Zn}]<-1)$. Our results in Table 7 and Figure 9 can also be used to check the variation in metal-line EW over a transverse physical scale of $\sim 0.7 \mathrm{kpc}$ (e.g., Koyamada et al. 2017; Rubin et al. 2018). Finally, we note that this work and a spectroscopic study of SDSS J1442+4055 by Krogager et al. (2018) have been conducted concurrently but independently. Krogager et al. (2018) used Keck spectra and data analysis methods different from ours to obtain results similar to those we present here.

We thank the anonymous referee for helpful comments that contributed to improving the final version of the paper. The Liverpool Telescope is operated on the island of La Palma by Liverpool John Moores University in the Spanish Observatorio del Roque de los Muchachos of the Instituto de Astrofisica de Canarias with financial support from the UK Science and Technology Facilities Council. This article is also based on observations made with the Gran Telescopio Canarias, installed at the Spanish Observatorio del Roque de los Muchachos of the Instituto de Astrofísica de Canarias on the island of La Palma. We thank the staff of both telescopes for kind interaction before, during, and after the observations. We also used data taken from the Sloan Digital Sky Survey (SDSS) database. The SDSS is managed by the Astrophysical Research Consortium for the Participating Institutions of the SDSS Collaboration. The SDSS website is www.sdss.org. Funding for the SDSS has been provided by the Alfred P. Sloan Foundation, the Participating Institutions, and national agencies in the U.S. and other countries. The SDSS acknowledges support and resources from the Center for High-Performance Computing at the University of Utah. We are grateful to the SDSS collaboration for doing that public database. This research has been conducted in the framework of the Gravitational LENses and DArk MAtter (GLENDAMA) project, which was/is supported by Spanish Department of Research, Development and Innovation grant AYA2013-47744C3-2-P; MINECO/AEI/FEDER-UE grant AYA2017-89815-P; the complementary action "Lentes Gravitatorias y Materia Oscura" financed by the SOciedad para el DEsarrollo Regional de
CANtabria (SODERCAN S.A.); the Operational Programme of FEDER-UE; and the University of Cantabria.

Facilities: Liverpool:2m(IO:O and SPRAT), GTC(OSIRIS).

Software: IRAF (http://iraf.noao.edu/), PyRAF (http://www. stsci.edu/institute/software_hardware/pyraf), SciPy (https:// www.scipy.org/), Astropy (Astropy Collaboration et al. 2013, 2018), Linetools (Prochaska et al. 2017), IMFITFITS (McLeod et al. 1998), VoigtFit (Krogager 2018), LENSMODEL (Keeton 2001).

\section{ORCID iDs}

Luis J. Goicoechea (1) https://orcid.org/0000-0003-0110-834X

\section{References}

Ahn, C. P., Alexandroff, R., Allende Prieto, C., et al. 2014, ApJS, 211, 17 Anguita, T., Schechter, P. L., Kuropatkin, N., et al. 2018, MNRAS, 480, 5017 Asplund, M., Grevesse, N., Sauval, A. J., \& Scott, P. 2009, ARA\&A, 47, 481 Astropy Collaboration, Price-Whelan, A. M., Sipőcz, B. M., et al. 2018, AJ, 156,123

Astropy Collaboration, Robitaille, T. P., Tollerud, E. J., et al. 2013, A\&A, 558, A33

Bonvin, V., Courbin, F., Suyu, S. H., et al. 2017, MNRAS, 465, 4914

Bruzual, A. G. 2003, in XI Canary Islands Winter School of Astrophysics, Galaxies at High Redshift, ed. I. Pérez-Fournon et al. (Cambridge: Cambridge Univ. Press), 185

Cardelli, J. A., Clayton, G. C., \& Mathis, J. S. 1989, ApJ, 345, 245

Cooke, R., Pettini, M., Steidel, C. C., et al. 2010, MNRAS, 409, 679

Dai, X., \& Kochanek, C. S. 2009, ApJ, 692, 677

Dawson, K. S., Schlegel, D. J., Ahn, C. P., et al. 2013, AJ, 145, A10

Draine, B. T. 2003, ARA\&A, 41, 241

Draine, B. T. 2011, Physics of the Interstellar and Intergalactic Medium (Princeton, NJ: Princeton Univ. Press)

Elíasdóttir, Á., Fynbo, J. P. U., Hjorth, J., et al. 2009, ApJ, 697, 1725

Elíasdóttir, Á., Hjorth, J., Toft, S., Burud, I., \& Paraficz, D. 2006, ApJS, 166,443

Ellison, S. L., Prochaska, J. X., Hennawi, J., et al. 2010, MNRAS, 406, 1435

Falco, E. E., Impey, C. D., Kochanek, C. S., et al. 1999, ApJ, 523, 617

Fall, S. M., \& Pei, Y. C. 1989, ApJ, 337, 7

Findlay, J. R., Prochaska, J. X., Hennawi, J. E., et al. 2018, ApJS, 236, 44

Fitzpatrick, E. L., \& Massa, D. 1990, ApJS, 72, 163

Fitzpatrick, E. L., \& Massa, D. 2007, ApJ, 663, 320

Gil-Merino, R., Goicoechea, L. J., Shalyapin, V. N., \& Oscoz, A. 2018, A\&A, 616, A118

Goicoechea, L. J., \& Shalyapin, V. N. 2016, A\&A, 596, A77

Gordon, K. D., Clayton, G. C., Misselt, K. A., Landolt, A. U., \& Wolff, M. J. 2003, ApJ, 594, 279

Hainline, L. J., Morgan, C. W., MacLeod, C. L., et al. 2013, ApJ, 774, 69 Hamuy, M., Suntzeff, N. B., Heathcote, S. R., et al. 1992, PASP, 104, 533

Hamuy, M., Suntzeff, N. B., Heathcote, S. R., et al. 1994, PASP, 106, 566

Horne, K. 1986, PASP, 98, 609

Howell, S. B. 2006, Handbook of CCD Astronomy (Cambridge: Cambridge Univ. Press)

Keeton, C. R. 2001, arXiv:astro-ph/0102340

Keeton, C. R. 2010, GReGr, 42, 2151

Kochanek, C. S. 2004, ApJ, 605, 58

Komatsu, E., Dunkley, J., Nolta, M. R., et al. 2009, ApJS, 180, 330

Kostrzewa-Rutkowska, Z., Kosłowski, S., Lemon, C., et al. 2018, MNRAS, 476, 663

Koyamada, S., Misawa, T., Inada, N., et al. 2017, ApJ, 851, A88

Krogager, J. K. 2018, VoigtFit: Absorption Line Fitting for Voigt Profiles, Astrophysics Source Code Library, ascl:1811/016

Krogager, J. K., Noterdaeme, P., O'Meara, J. M., et al. 2018, A\&A, 619, A142

Ledoux, C., Noterdaeme, P., Petitjean, P., \& Srianand, R. 2015, A\&A, 580, A8

Lemon, C. A., Auger, M. W., McMahon, R. G., \& Ostrovski, F. 2018, MNRAS, 479, 5060

Liszt, H. 2014, ApJ, 783, A17

Lusso, E., Fumagalli, M., Rafelski, M., et al. 2018, ApJ, 860, A41

Ma, J., Ge, J., Prochaska, J. X., et al. 2018, MNRAS, 474, 4870

Ma, J., Ge, J., Zhao, Y., et al. 2017, MNRAS, 472, 2196

McLeod, B. A., Bernstein, G. M., Rieke, M. J., \& Weedman, D. W. 1998, AJ, 115,1377

Mediavilla, E., Muñoz, J. A., Kochanek, C. S., et al. 2005, ApJ, 619, 749 
Mediavilla, E., Muñoz, J. A., Kochanek, C. S., et al. 2011, ApJ, 730, 16

Ménard, B., \& Chelouche, D. 2009, MNRAS, 393, 808

Møller, P., Fynbo, J. P. U., Ledoux, C., \& Nilsson, K. K. 2013, MNRAS, 430, 2680

More, A., Oguri, M., Kayo, I., et al. 2016, MNRAS, 456, 1595

Oke, J. B. 1990, AJ, 99, 1621

Østman, L., Goobar, A., \& Mörtsell, E. 2008, A\&A, 485, 403

Pâris, I., Petitjean, P., Aubourg, É., et al. 2014, A\&A, 563, A54

Pâris, I., Petitjean, P., Aubourg, É., et al. 2018, A\&A, 613, A51

Pelt, J., Kayser, R., Refsdal, S., \& Schramm, T. 1996, A\&A, 305, 97

Prochaska, J. X., Tejos, N., Crighton, N., et al. 2017, Linetools/linetools: Third Minor Release, Zenodo, doi:10.5281/zenodo.1036773

Rubin, K. H. R., O’Meara, J. M., Cooksey, K. L., et al. 2018, ApJ, 859, A146

Schneider, P., Kochanek, C. S., \& Wambsganss, J. 2006, in Proc. 33rd SaasFee Advanced Course, Gravitational Lensing: Strong, Weak \& Micro, ed. G. Meylan, P. Jetzer, \& P. North (Berlin: Springer)
Sergeyev, A. V., Zheleznyak, A. P., Shalyapin, V. N., \& Goicoechea, L. J. 2016, MNRAS, 456, 1948

Shalyapin, V. N., \& Goicoechea, L. J. 2017, ApJ, 836, A14

Sluse, D., Claeskens, J. F., Hutsemékers, D., \& Surdej, J. 2007, A\&A, 468, 885

Sluse, D., Schmidt, R., Courbin, F., et al. 2011, A\&A, 528, A100

Smette, A., Surdej, J., Shaver, P. A., et al. 1992, ApJ, 389, 39

Tewes, M., Courbin, F., \& Meylan, G. 2013, A\&A, 553, 120

Tie, S. S., \& Kochanek, C. S. 2018, MNRAS, 473, 80

Treu, T. 2010, ARA\&A, 48, 87

Ullán, A., Goicoechea, L. J., Zheleznyak, A. P., et al. 2006, A\&A, 452, 25

Vladilo, G., Prochaska, J. X., \& Wolfe, A. M. 2008, A\&A, 478, 701

Vuissoz, C., Courbin, F., Sluse, D., et al. 2008, A\&A, 488, 481

Wolfe, A. M., Turnshek, D. A., Smith, H. E., \& Cohen, R. D. 1986, ApJS, 61,249

Wucknitz, O., Wisotzki, L., López, S., \& Gregg, M. D. 2003, A\&A, 405, 445

York, D. G., Adelman, J., Anderson, J. E., et al. 2000, AJ, 120, 1579

Zuo, L., Beaver, E. A., Burbidge, E. M., et al. 1997, ApJ, 477, 568 